\title{
Packaged Capacitive Pressure Sensor System for Aircraft Engine Health Monitoring
}

Dr. Maximilian C. Scardelletti - NASA Glenn Research Center Dr. Christian A. Zorman - Case Western Reserve University 


\section{Introduction}

- Sensing systems for harsh environments:

High temperature electronics and sensors

Three Major Industries

- Downhole Oil and Gas

Drilling operations were limited 150 to $175^{\circ} \mathrm{C}$ for reserves in easily accessible wells

Declining reserves force deeper wells, which increase drilling temperatures to $300^{\circ} \mathrm{C}$

- Automobile

Cylinder pressures temperature: $300^{\circ} \mathrm{C}$

Exhaust sensing temperature: $850^{\circ} \mathrm{C}$

- Aerospace

Monitoring the health of aircraft engines at temperatures above $300^{\circ} \mathrm{C}$ (emissions, temperature, blade tip clearance and pressure) Atmospheric and surface conditions of Venus $\left(480^{\circ} \mathrm{C}\right)$ 


\section{Introduction}

Develop a SiC-based MEMs capacitive pressure sensor system that can be used to monitor the pressure of a conventional gas turbofan engine.

Operating Conditions: Temperature: 25 to $500^{\circ} \mathrm{C}$ Pressure: 0 to $300 \mathrm{psi}$ Vibration: up to $5.3 \mathrm{G}_{\mathrm{rms}}$ 


\section{System Design}

The system is realized by integrating the following components on a common, high temperature substrate

1. A novel SiCN MEMS capacitive pressure sensor

2. $6 \mathrm{H}-\mathrm{SiC}$ MESFET as active device

3. MIM capacitors, wirewound inductors, thick film resistors

4. Low form factor packaging

5. Borescope plug adaptor 


\section{Electronics Design}

\section{The proposed system uses a Clapp-Type Oscillator Design}

- $\quad$ The integrated system uses a Clapp-type oscillator with capacitive pressure sensor located in LC tank circuit

- As pressure increases, pressure sensor capacitance decreases, which causes the operational frequency to increase

- $\quad$ Cree SiC MESFET used for driving circuit into oscillation

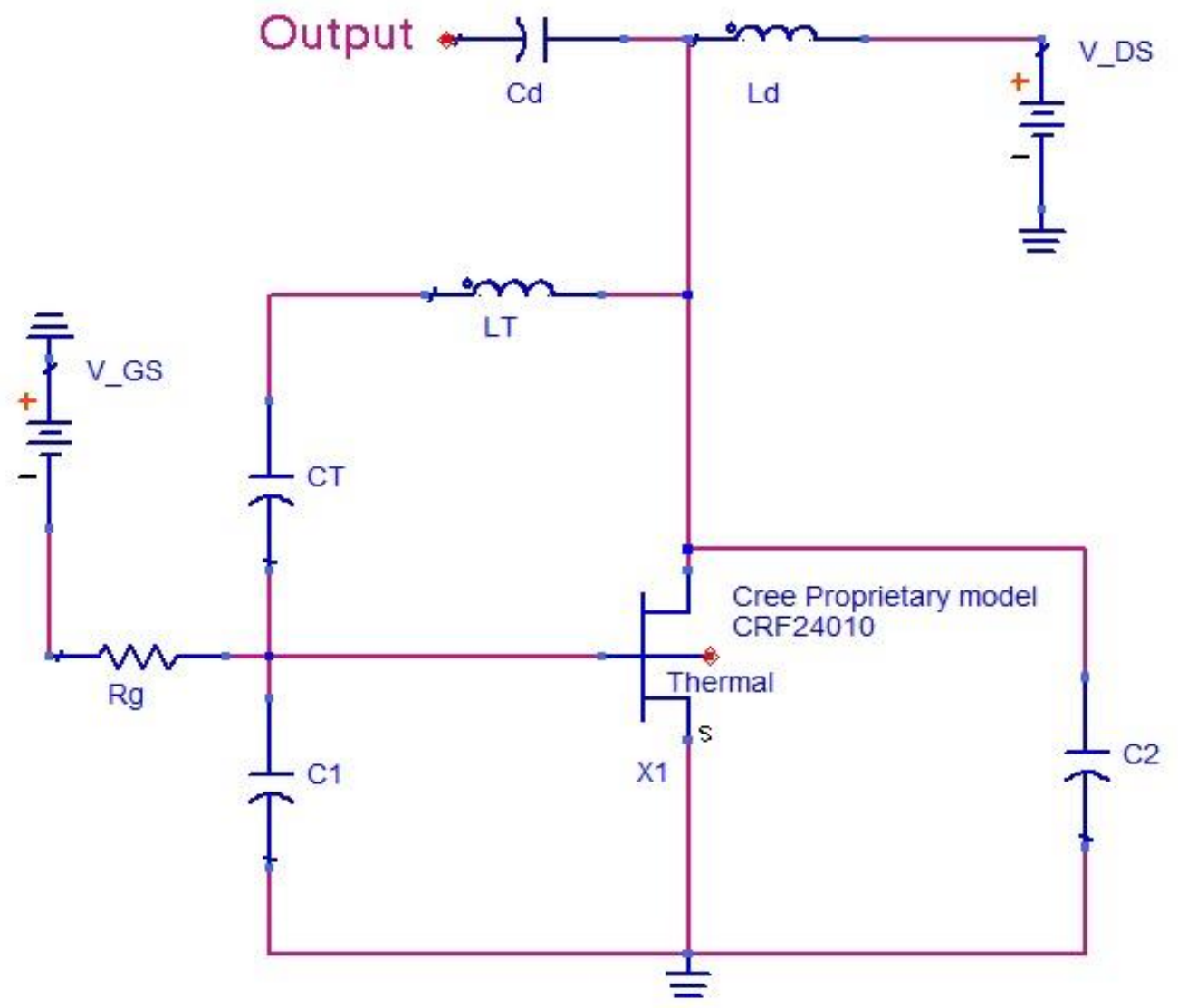




\section{Electronics Design}

\section{Clapp-Type Oscillator vs Colpitts Oscillator}

- The proposed Clapp oscillator requires one inductor, three capacitors and one MESFET. Requires fewer components vs. Colpitts oscillator design

- Increases system efficiency

- Increases system reliability under harsh environment conditions

- $\mathrm{L}_{\mathrm{T}}$ and $\mathrm{C}_{\mathrm{SENSE}}$ are in series and $\mathrm{C}_{\mathrm{SENSE}}$ is used to set the operational frequency

- $\mathrm{C}_{1}$ and $\mathrm{C}_{2}$ are used to control the gain conditions

- This arrangement increases frequency stability, making it more frequency stable than the Colpitts design. 


\section{Pressure Sensor Testing}

High Temperature and Pressure Chamber

System Key Features

- Pressure range: 0 to $100 \mathrm{psi}$

- Temperature range: 25 to $500^{\circ} \mathrm{C}$

- LabVIEW control program

- Power source

- Multiple thermocouple

- Multiple feedthroughs
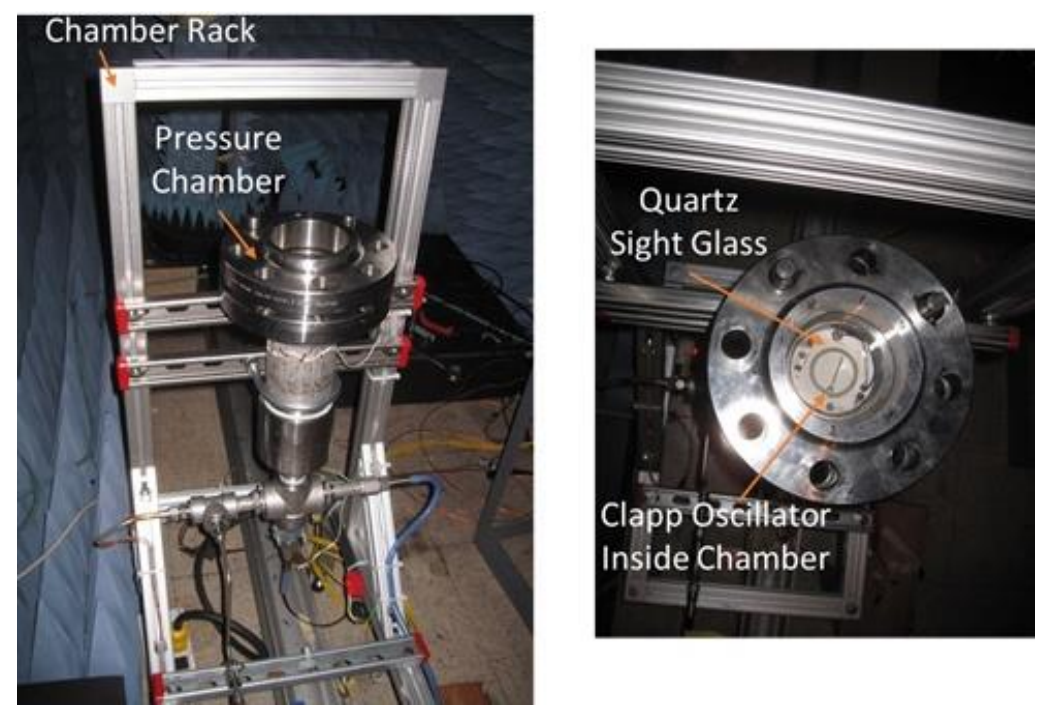

- Sight glass for signal transmission 


\section{Pressure Sensor}

\section{Sporian SiCN Capacitive Pressure Sensor}

- Parallel plate capacitor model

- SiCN membrane

- Temperature range: up to $1000^{\circ} \mathrm{C}$

\section{Pressure Capacitance} (psi)

0

50

SiCN Capacitive

Pressure Sensor

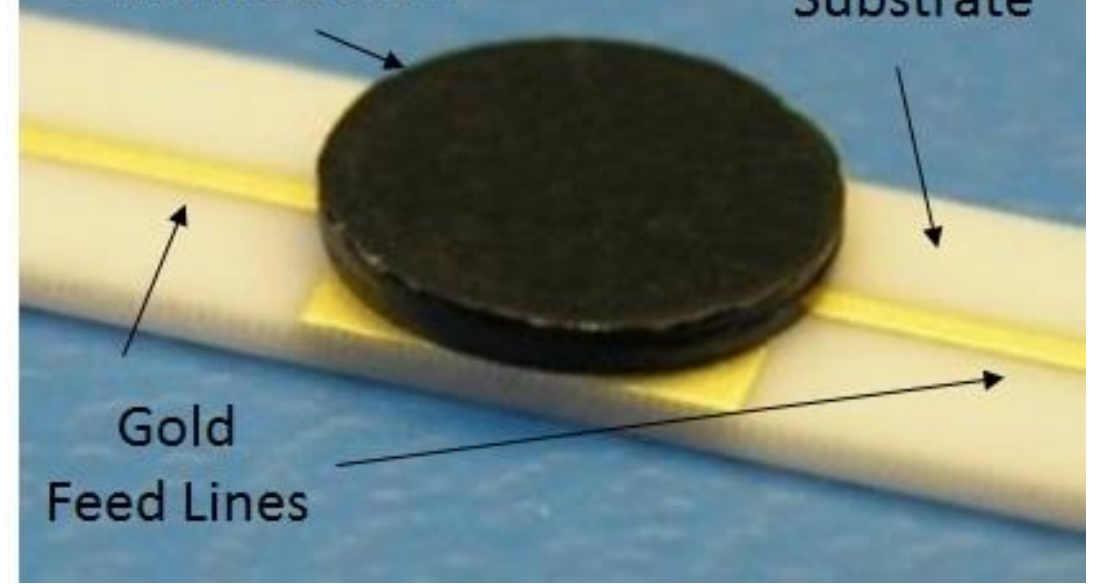

SiCN pressure sensor
Alumina

Substrate (a)

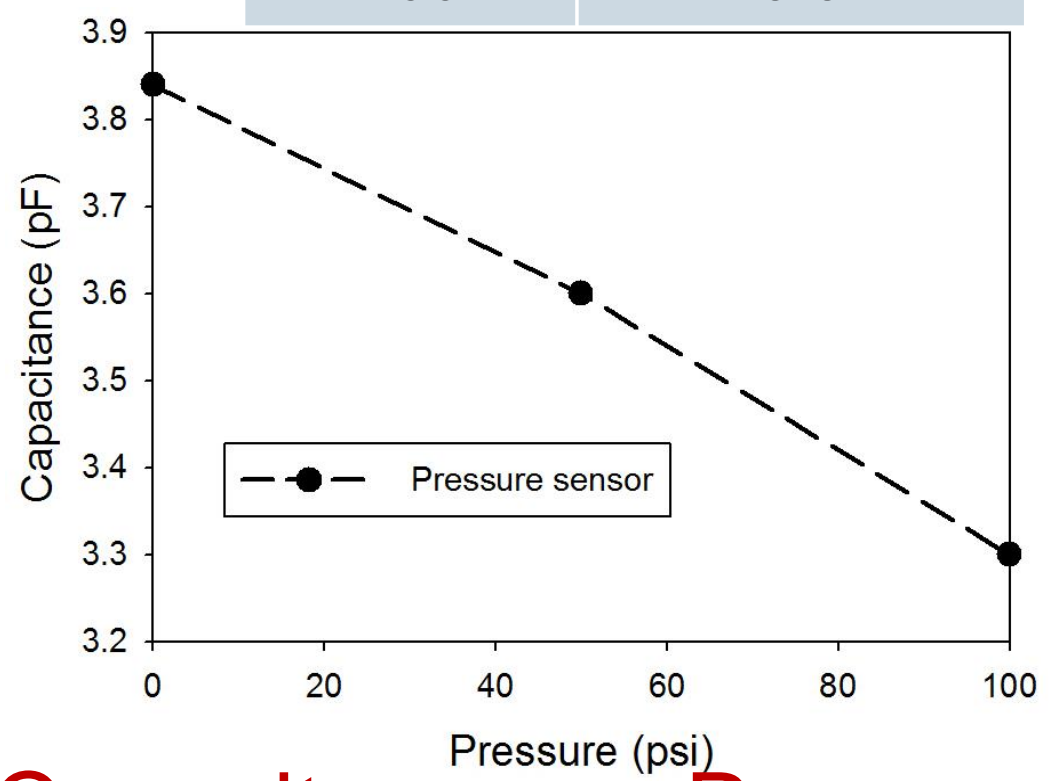

Capacitance vs Pressure 


\section{Oscillator Design}

\section{Circuit Simulations}

\section{Keysight's Advanced Design System (ADS) Software suite}

$$
\begin{aligned}
& \mathrm{CT}=3.84 \mathrm{pF} \\
& \mathrm{LT}=780 \mathrm{nH} \\
& \mathrm{C} 1=14 \mathrm{pF} \\
& \mathrm{C} 2=41 \mathrm{pF} \\
& \mathrm{RG}=10 \mathrm{~K} \Omega \\
& \mathrm{LD}=390 \mathrm{nH} \\
& \mathrm{CD}=188 \mathrm{pF}
\end{aligned}
$$$$
\text { Cree SiC MESFET model }
$$

\section{Oscillation Frequency 96.7 MHz}




\section{Oscillator Design}

\section{Circuit Simulations}
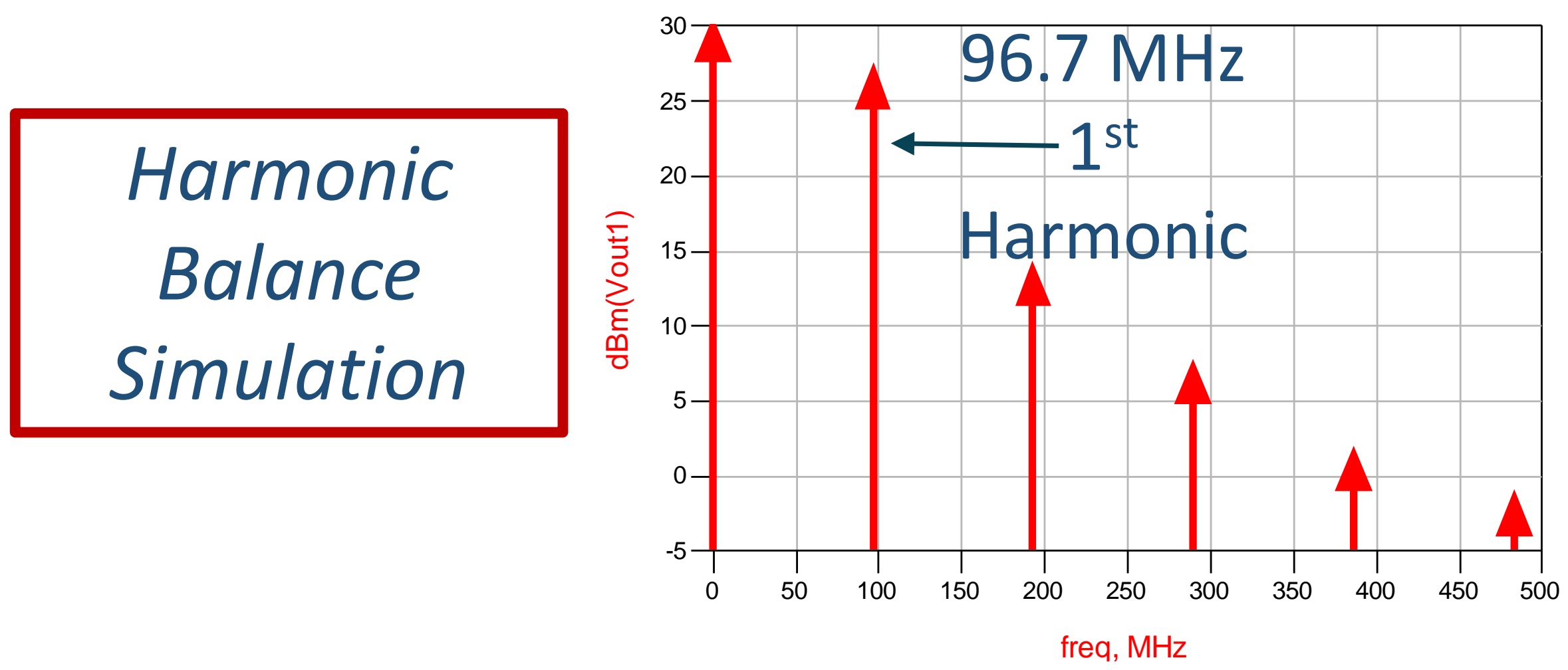


\section{Oscillator Design}

\section{Circuit Simulations}

To achieve oscillation stability

1) Phase must be zero at $f_{o}$

2) Loop gain must be greater than unity at $f_{o}$
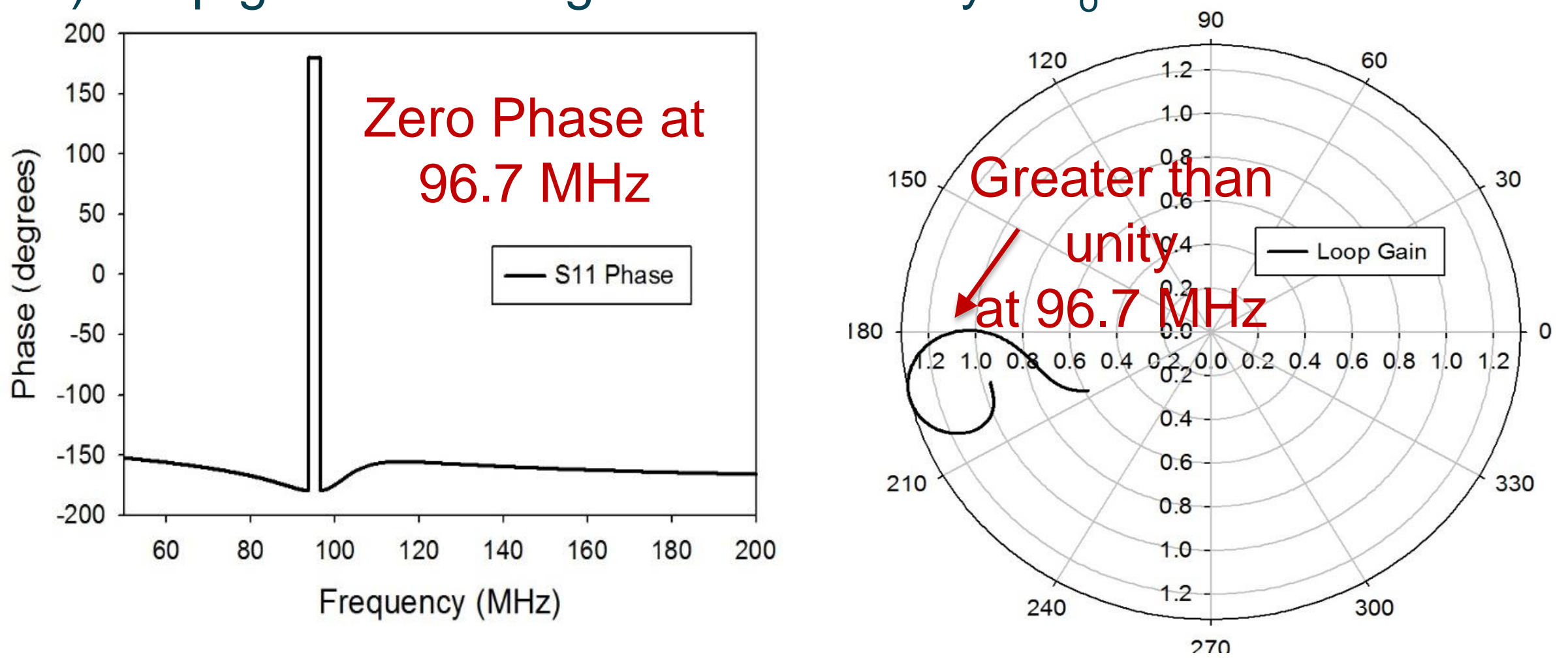


\section{Oscillator Design}

\section{Circuit Simulations}

\section{Harmonic} Balance Simulation

$\mathrm{P}=0$ psi $\mathrm{f}=96.7 \mathrm{MHz}$

$\mathrm{P}=50$ psi $f=99.2 \mathrm{MHz}$

$\mathrm{P}=100 \mathrm{psi} f=102.8 \mathrm{MHz}$

$$
\mathrm{P}=0 \text { psi } \quad \mathrm{P}=50 \text { psi } \mathrm{P}=100 \text { psi }
$$

$$
\mathrm{C}=3.84 \mathrm{pF} \quad \mathrm{C}=3.6 \mathrm{pF} \quad \mathrm{C}=3.3 \mathrm{pF}
$$

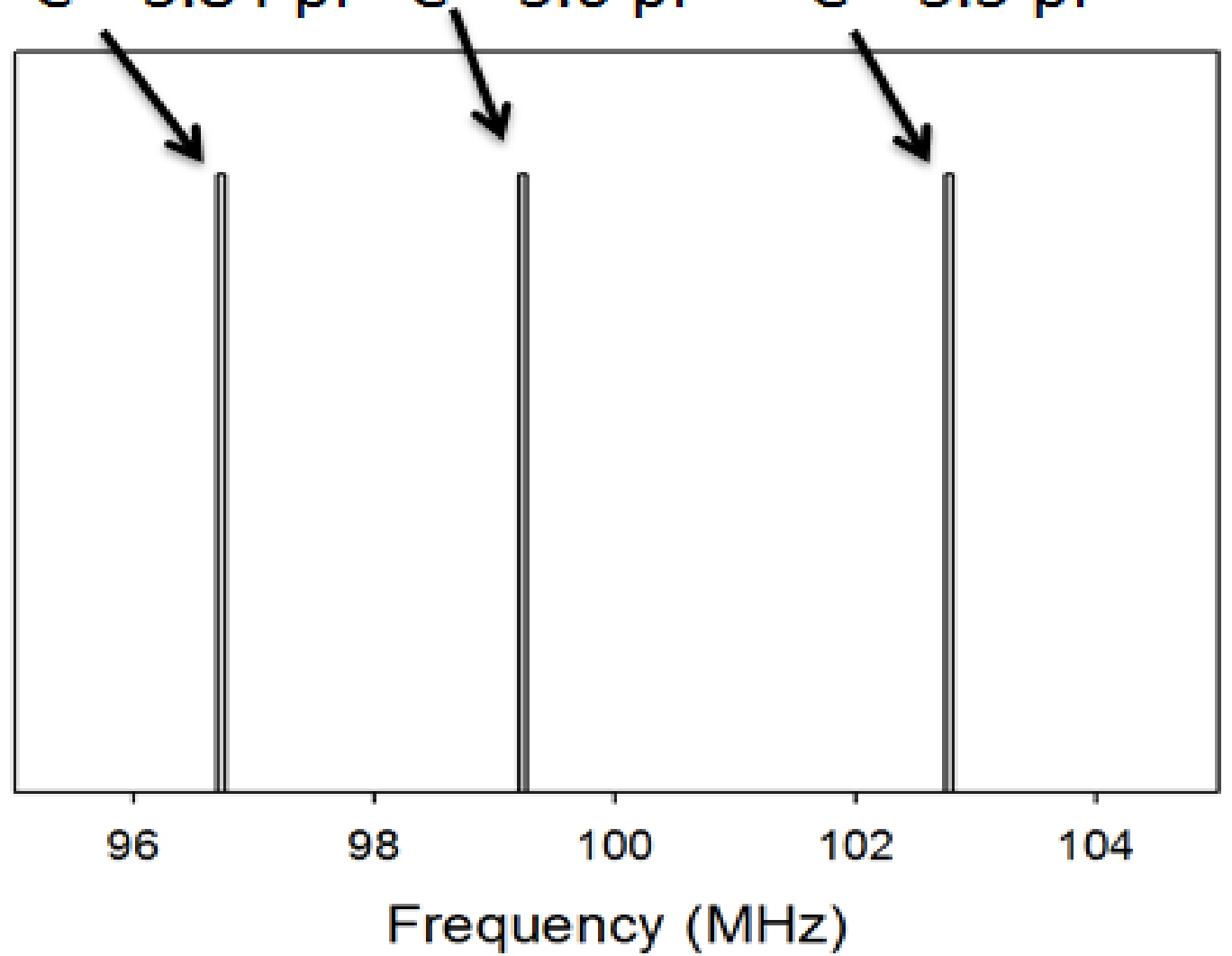




\section{Engine Testing}

\section{Packaged wired prototype has the following}

characteristics

- Unpackaged Sensor System Size: 8 x 40 × 4 mm (including on-board DC bias circuits)

- Form Factor: Packaged sensor equipped with borescope adaptor for a borescope plug on engine

- Maximum Operational Temperature: $500^{\circ} \mathrm{C}$ for 1 hour at tip of borescope adaptor

- Maximum Vibration: $5.3 G_{r m s}$ along $X-, Y$ - and Zaxis for 20 min 


\section{Pressure Sensor System}

Entire circuit assembled on a single alumina substrate

$\left(6 \times 35 \times 2 \mathrm{~mm}^{3}\right)$

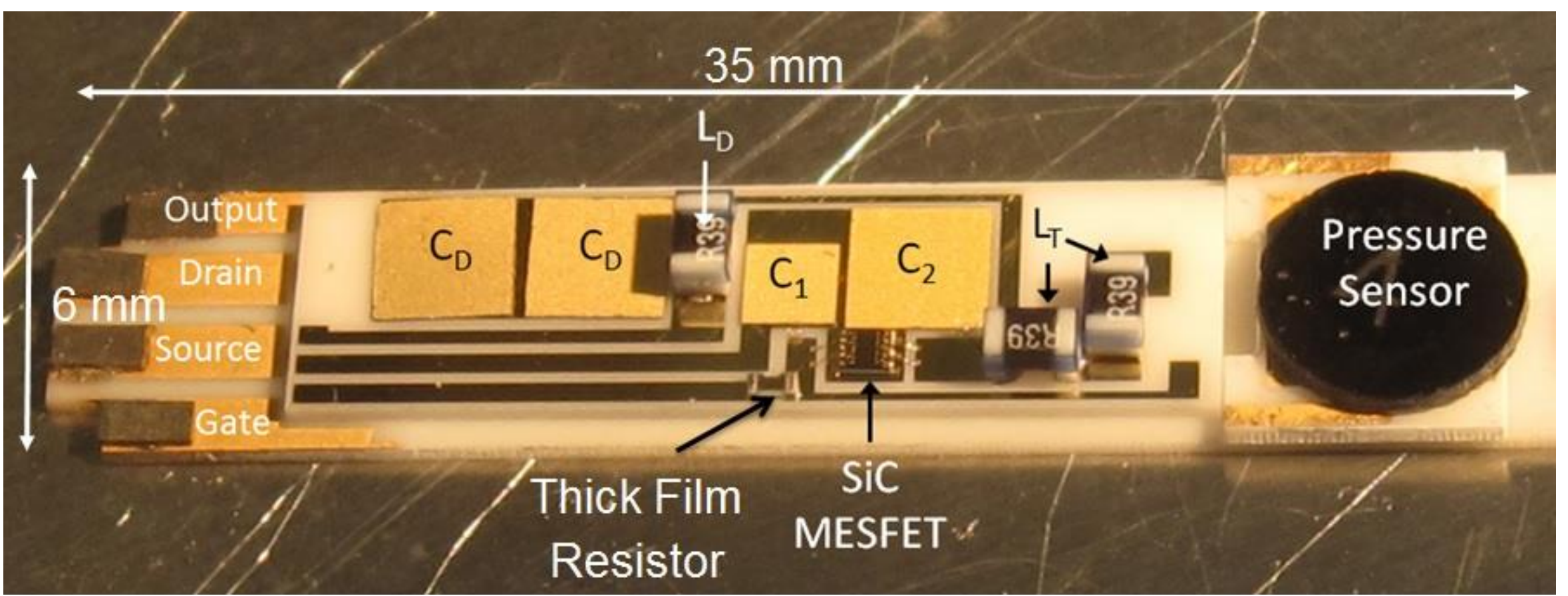




\section{Pressure Sensor System}

\section{Packaged Sensor System Assembly}

\section{Key Features}

- Stainless steal packaging

- Thermo couples

- Custom connector/cable from package to facilitate input power and output signal

- Borescope plug adaptor

- Size: 30 x $150 \mathrm{~mm}$ 


\section{Pressure Sensor System}

\section{Bench-Top Acceptance Testing}

- Custom-in-house pressurized fixture

- Packaged sensor is attached to quasi-borescope adaptor

- Thermocouple inside fixture to emulate inner engine temperature

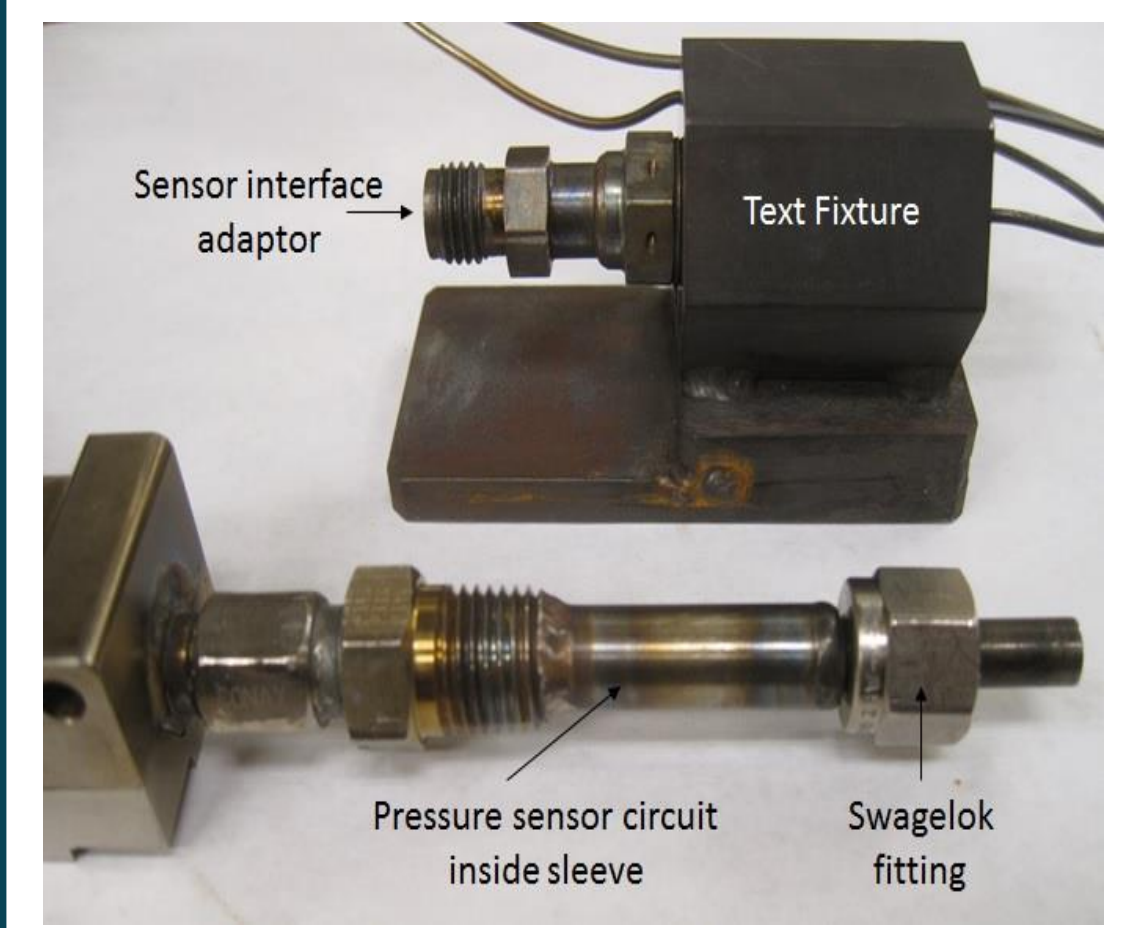




\section{Pressure Sensor System}

Bench-Top Packaged System Characterization

To emulate actual jet turbofan engine conditions the packaged sensor was heated to over $500^{\circ} \mathrm{C}$ and the pressure was increased from 0 to $300 \mathrm{psi}$.

Note: The temperature recorded on the metal sleeve was $\approx 400^{\circ} \mathrm{C}$, which is assumed to be the steady-state temperature of the

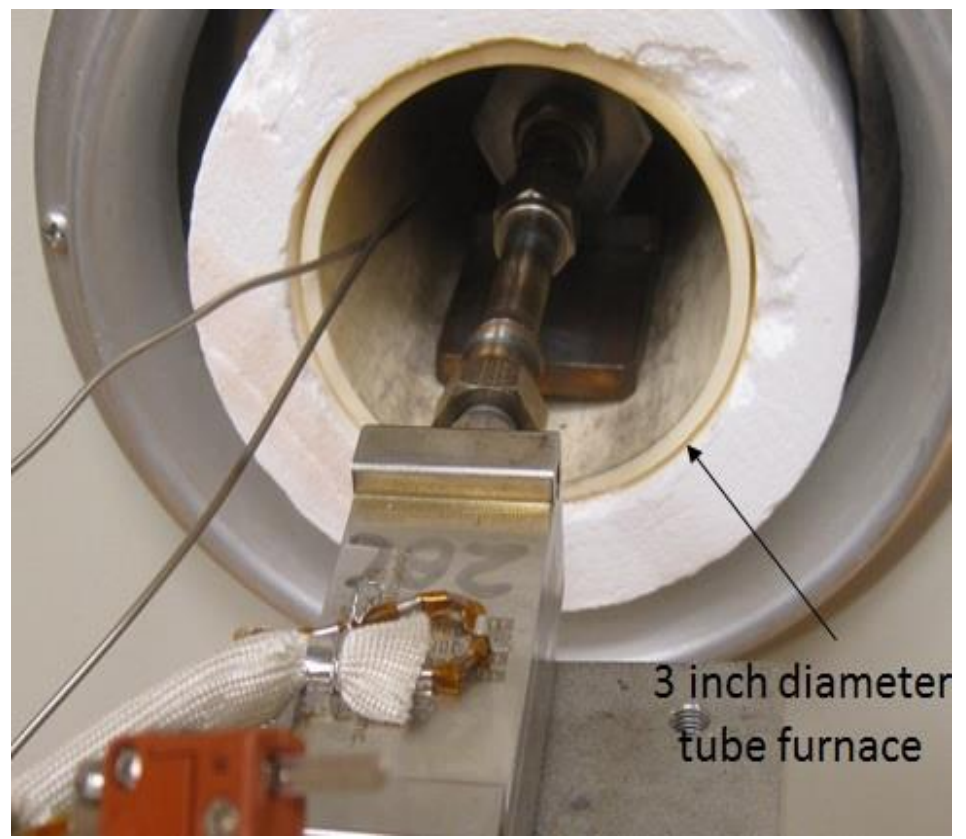
system 


\section{Pressure Sensor System}

\section{Bench-Top Packaged System Characterization}
0 psi $\rightarrow 96.88 \mathrm{MHz}$
$100 \mathrm{psi} \rightarrow 102.79 \mathrm{MHz}$ $200 \mathrm{psi} \rightarrow 109.54 \mathrm{MHz}$ $300 \mathrm{psi} \rightarrow 116.77 \mathrm{MHz}$ $350 \mathrm{psi} \rightarrow 119.86 \mathrm{MHz}$

Note: Simulated and measured response at 0 and 100 psi are virtually ${ }_{95}^{-100}$ identical: Incredibly accurate circuit model

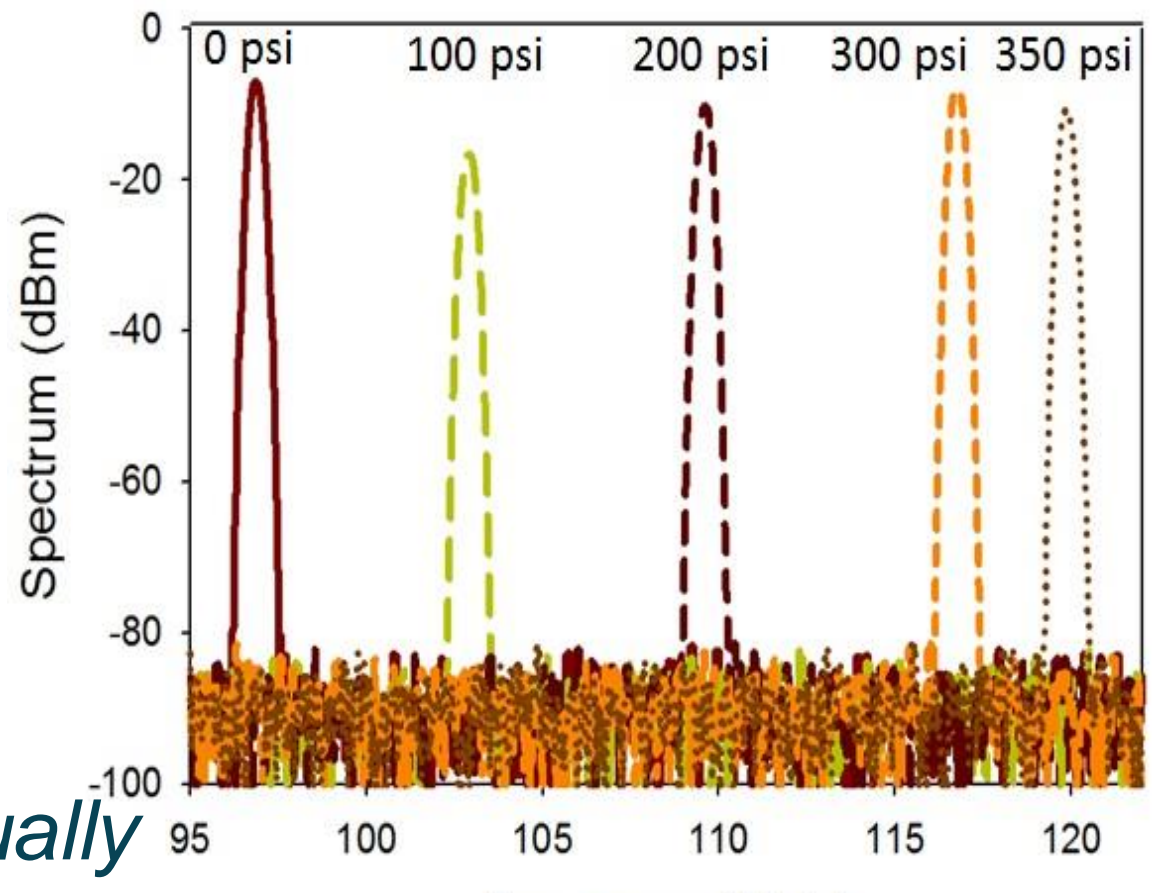

Frequency $(\mathrm{MHz})$
$6.57 \times 10^{-2} \Delta \mathrm{f} / \Delta \mathrm{P} \mathrm{MHz} / \mathrm{psi}$ Percent difference $=21.2 \%$
Spectrum response of packaged pressure sensor from 0 to 350 psi at $25^{\circ} \mathrm{C}$ 


\section{Pressure Sensor System}

\section{Bench-Top System Characterization}

The temperature at the tip of the sensor inside the pressurized is fixture is $540^{\circ} \mathrm{C}$

$\left(\approx 400^{\circ} \mathrm{C}\right.$ at the sleeve $)$

0 psi $\rightarrow 96.3 \mathrm{MHz}$ 320 psi $\rightarrow 117.8 \mathrm{MHz}$

$6.8 \times 10^{-2} \Delta \mathrm{f} / \Delta \mathrm{P} \mathrm{MHz} / \mathrm{psi}$ Percent difference $=20 \%$

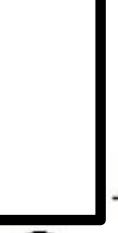

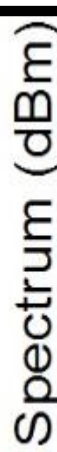

政 $-100$ (1)
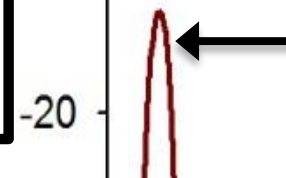

$$
\Delta f=21.5 \mathrm{MHz}
$$

$P=0$ psiat $540 \mathrm{C}$
$P=320$ psi at $540 \mathrm{C}$

\section{haracterization
The change in frequency
at 25 and $540^{\circ} \mathrm{C}$ at 0 psi is \\ haracterization
The change in frequency
at 25 and $540^{\circ} \mathrm{C}$ at 0 psi is less $1 \%$
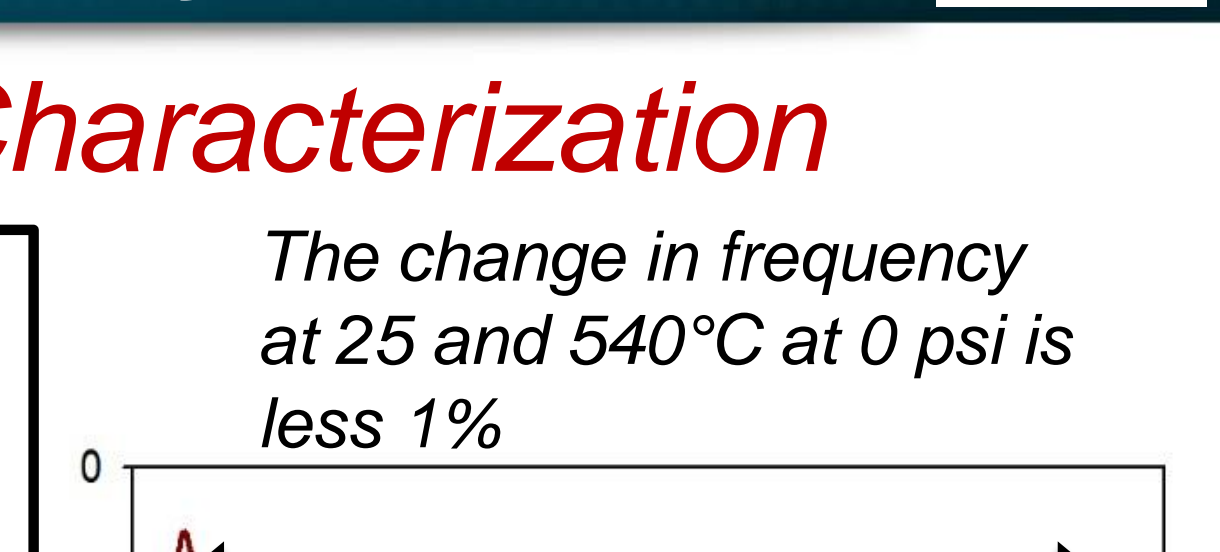

0

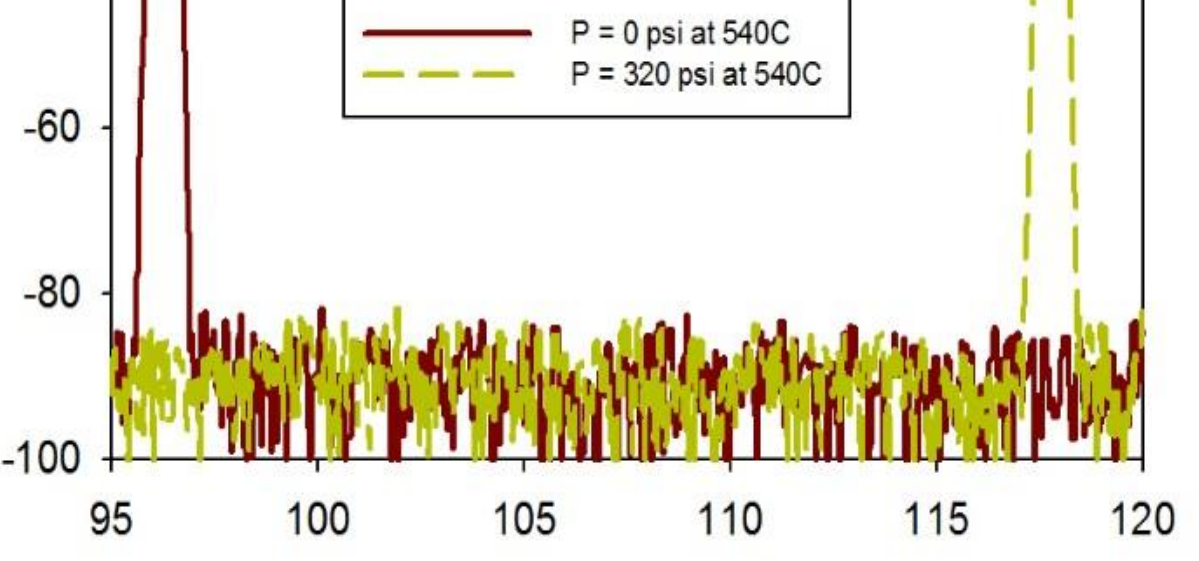

Frequency $(\mathrm{MHz})$ 


\section{Pressure Sensor System}

Bench-Top Packaged System Characterization

Structural Dynamic Testing

- Emulate on engine testing

- Sine wave sweeps

- Random vibration

- Maximum vibration $5.3 \mathrm{G}_{\mathrm{rms}}$

- $\quad \mathrm{X}$-, Y- and Z-axis testing

- Resonate frequency recorded at the beginning and end of each axis test. NO change!!

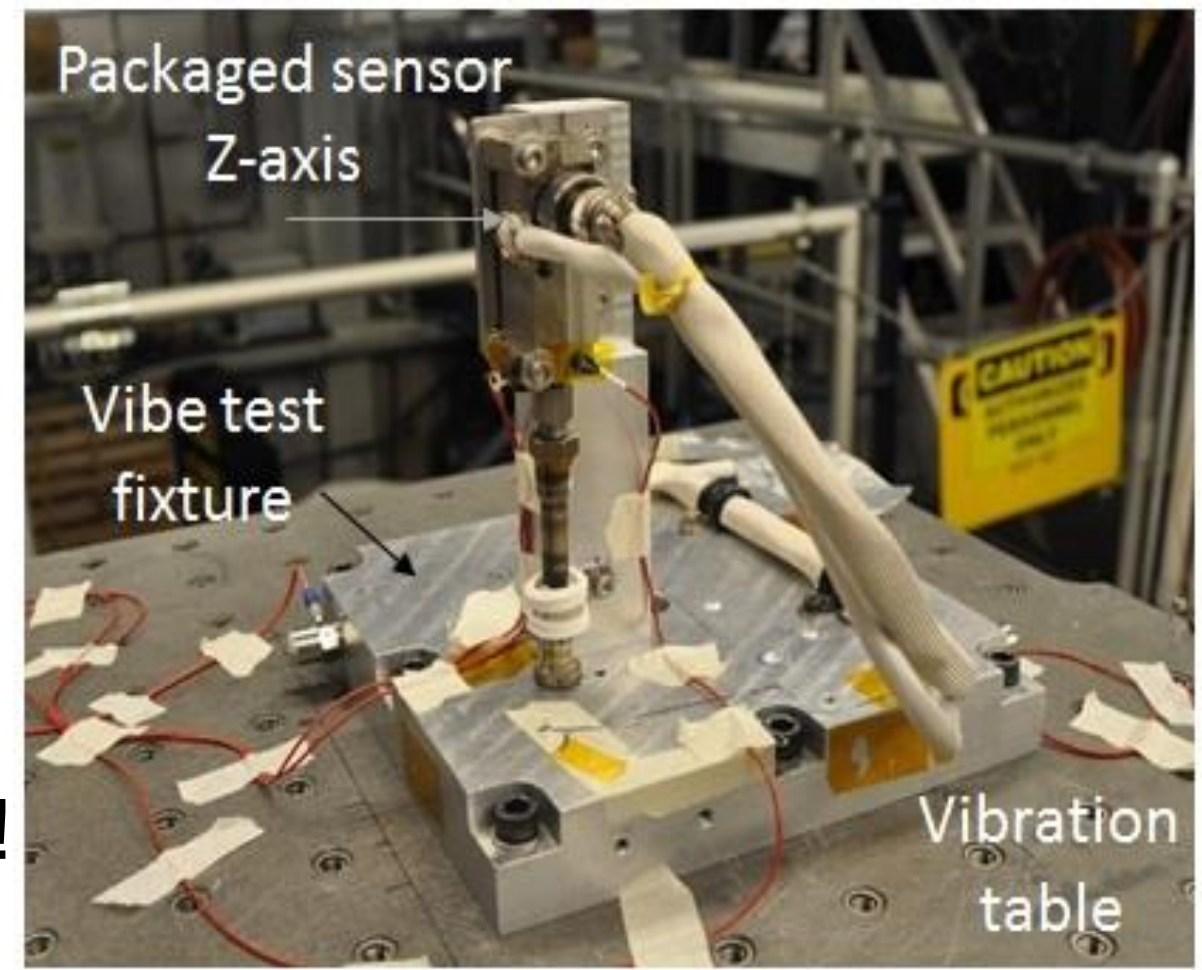




\section{Pressure Sensor System}

\section{Bench-Top Packaged System Characterization}

The packaged sensor system

was again measured after

structural dynamic testing.

$0 \mathrm{psi} \rightarrow 25^{\circ} \mathrm{C} \rightarrow 97.3 \mathrm{MHz}$

0 psi $\rightarrow 520^{\circ} \mathrm{C} \rightarrow 96.5 \mathrm{MHz}$

$342 \mathrm{psi} \rightarrow 520^{\circ} \mathrm{C} \rightarrow 118.1 \mathrm{MHz}$

The change in frequency at 25 and $540^{\circ} \mathrm{C}$ at 0 psi is less $1 \%$

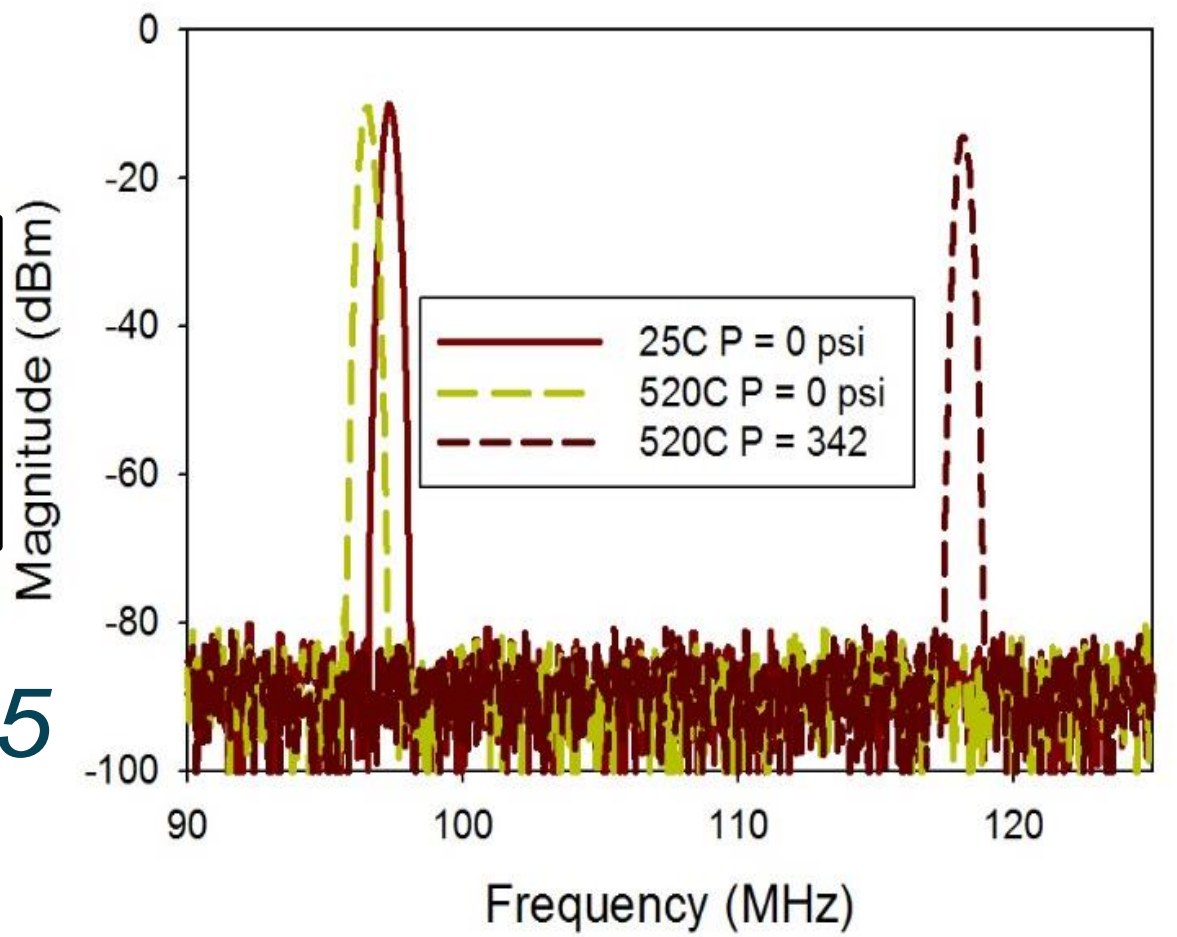




\section{Vehicle Integrated Propulsion (VIPR)}

VIPR was a series of ground-based onwing engine demonstrations to mature aircraft engine health management technologies

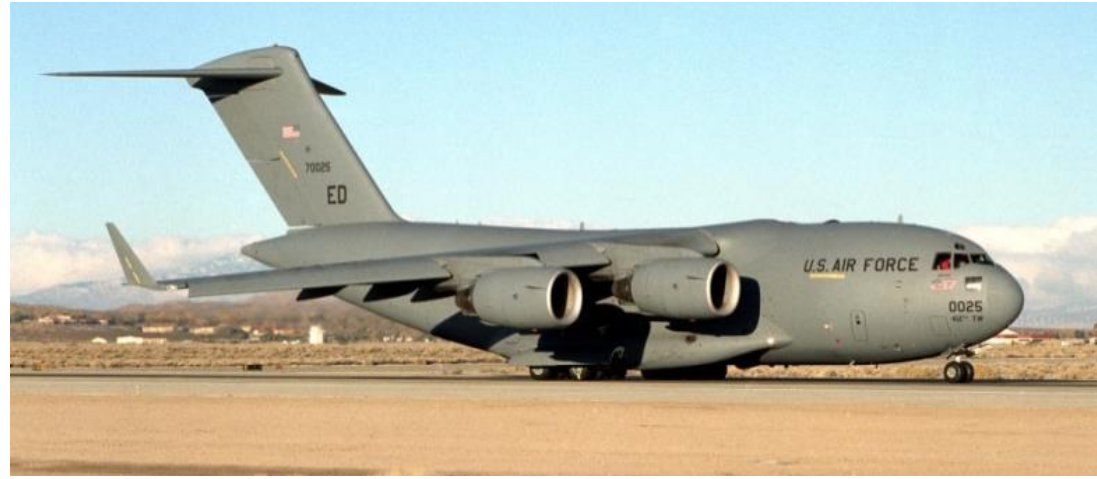

Test vehicle was a U.S. Air Force C-17 aircraft equipped with Pratt \& Whitney F117 engines

VIPR partners include NASA, U.S. Air Force, Pratt \& Whitney, GE, Rolls Royce, Boeing, FAA, USGS, and other external organizations

Boeing C-17 Globemaster III

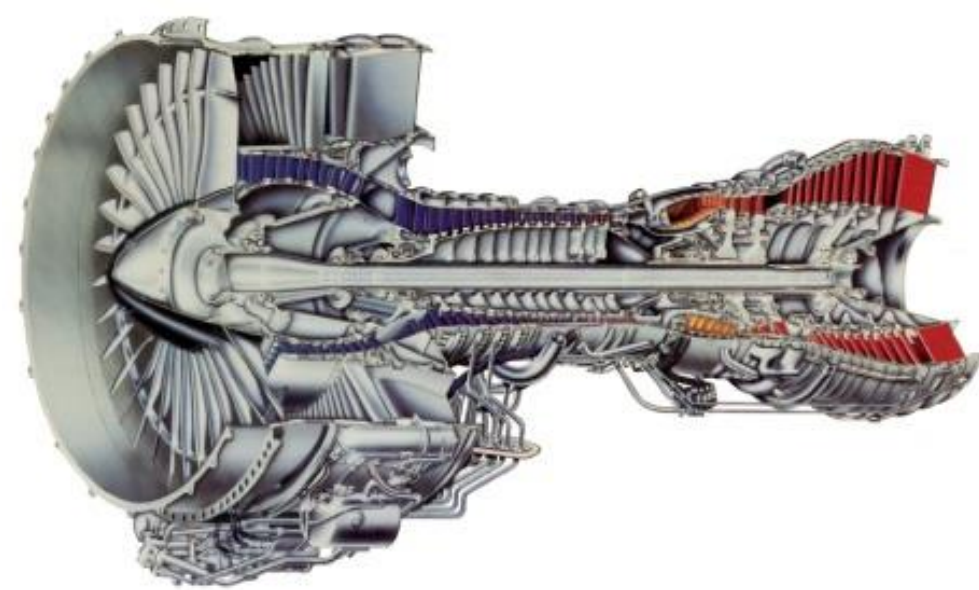

Pratt \& Whitney F117 Turbofan Engine 


\section{VIPR3}

Test Objectives:

Demonstrate capability of advanced health management technologies for detecting and diagnosing incipient engine faults before they become a safety impact and to minimize loss of capability

Self Diagnostic Accelerometer
Approach:

Perform on wing engine ground tests

- Normal engine operations

- Seeded mechanical faults

- Seeded gas path faults

- Accelerated engine life degradation through volcanic ash ingestion testing 


\section{VIPR3}

\section{CST Aircraft / Communication Layout}

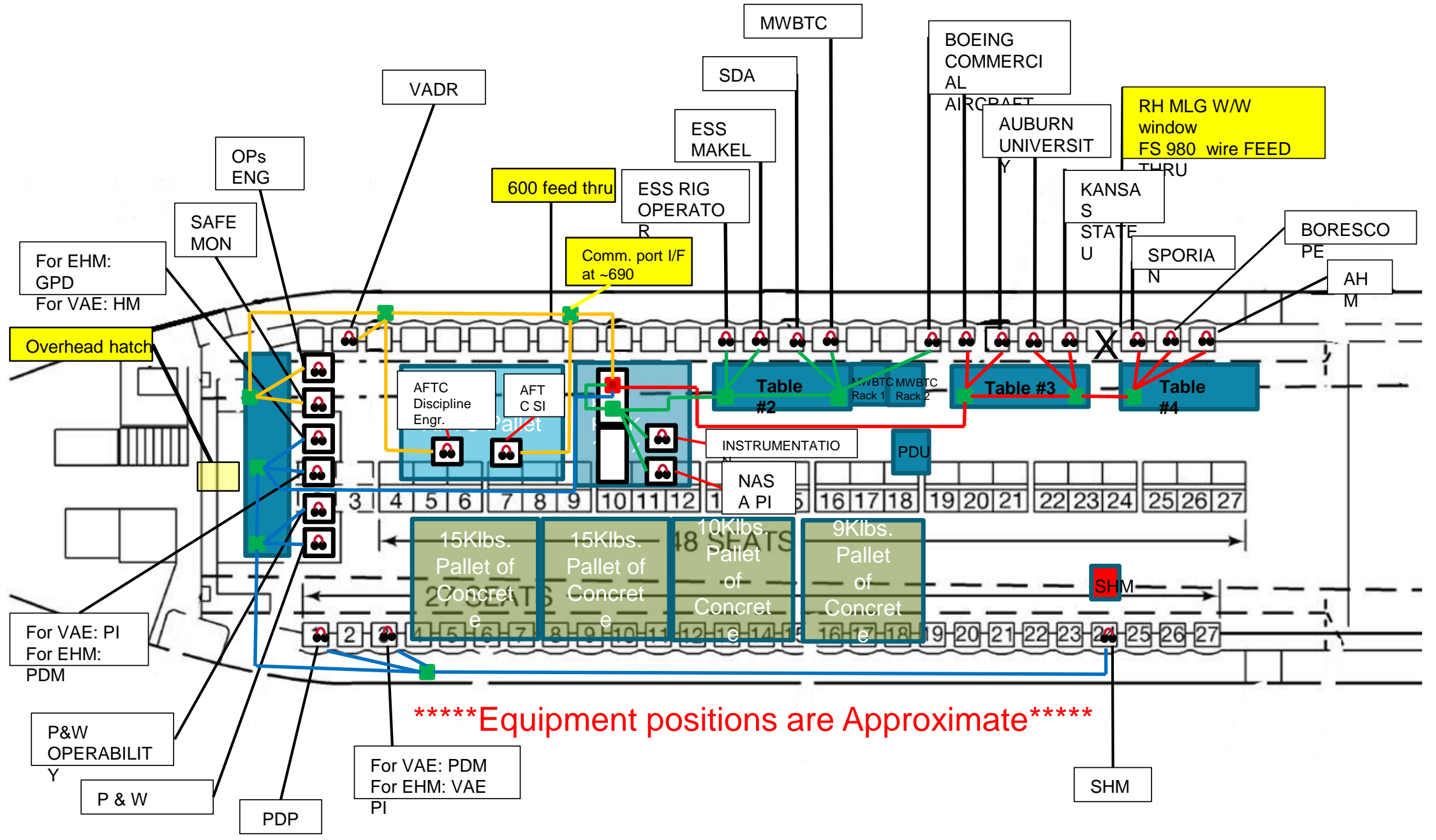




\section{VIPR3}

\section{Aircraft Research Station Layout}

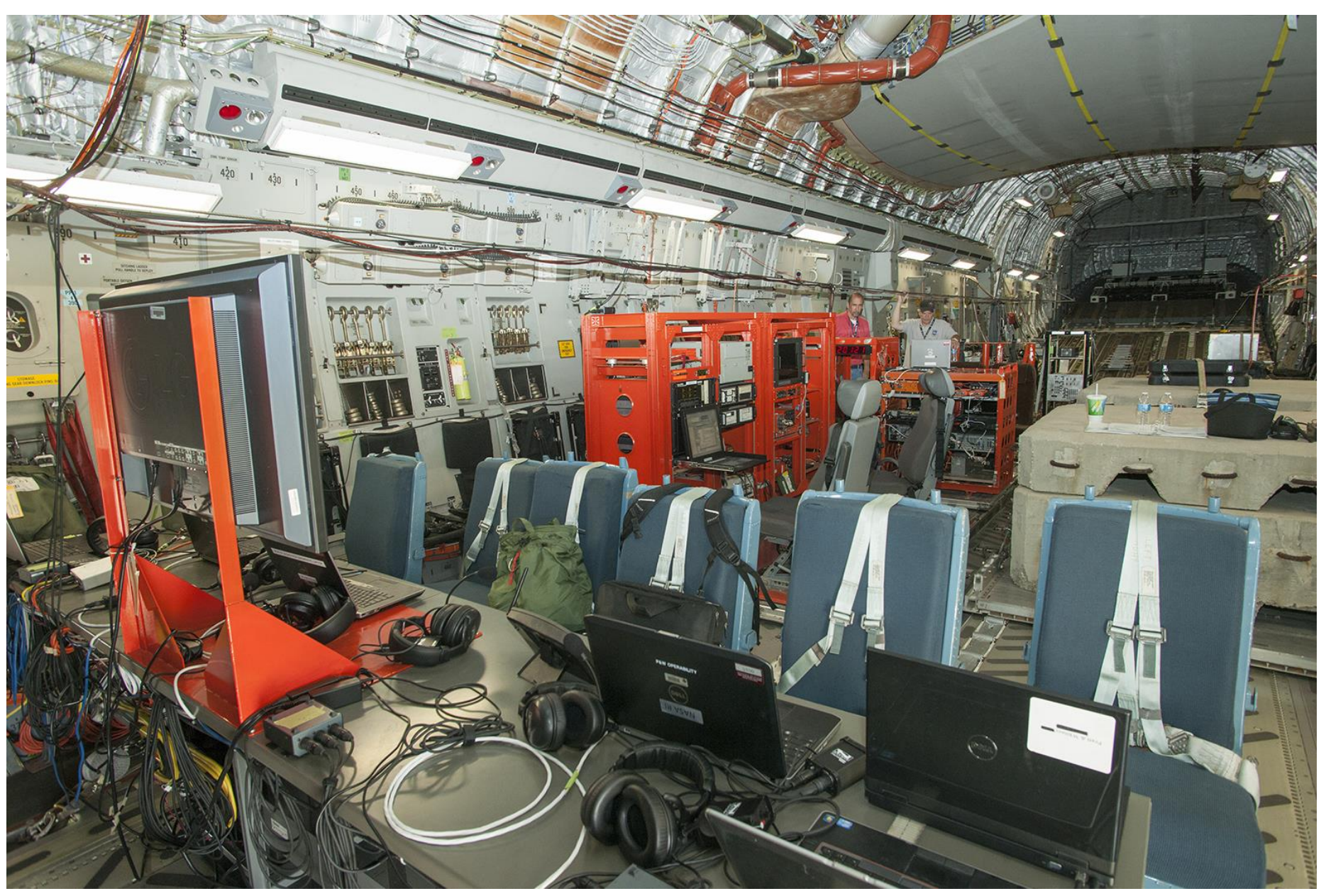




\section{VIPR3}

\section{Aircraft Research Station Layout}

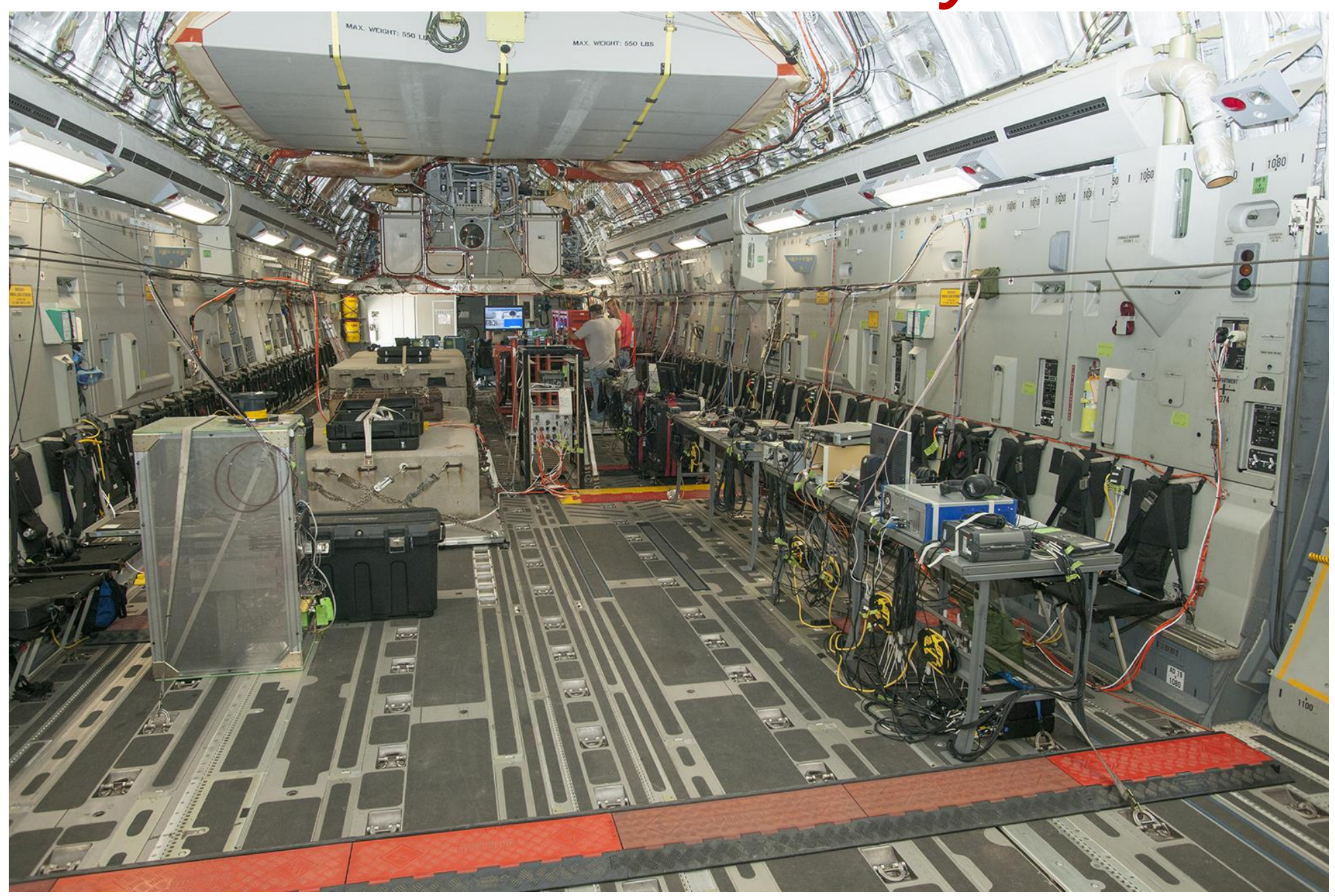




\section{VIPR3}

\section{Measurement setup in fuselage to sensor on the engine attached to the wing}

- Spectrum analyzer

- Power supply

- Laptop

- Labview program to record measurements

- $200 \mathrm{ft}$ cable going from equipment to sensor on engine

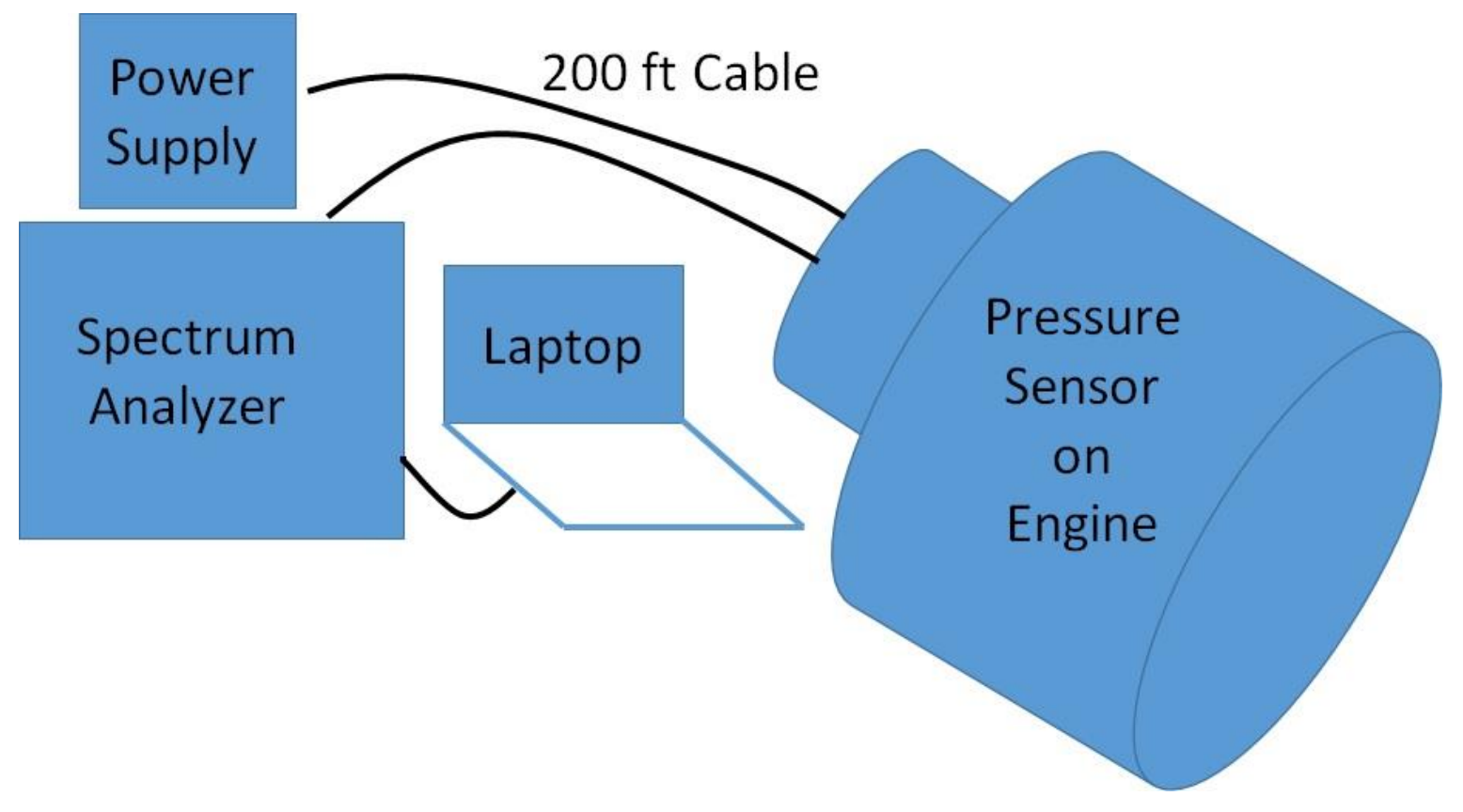




\section{Vehicle Integrated Propulsion (VIPR)}

\section{Sensed Pressure Locations}

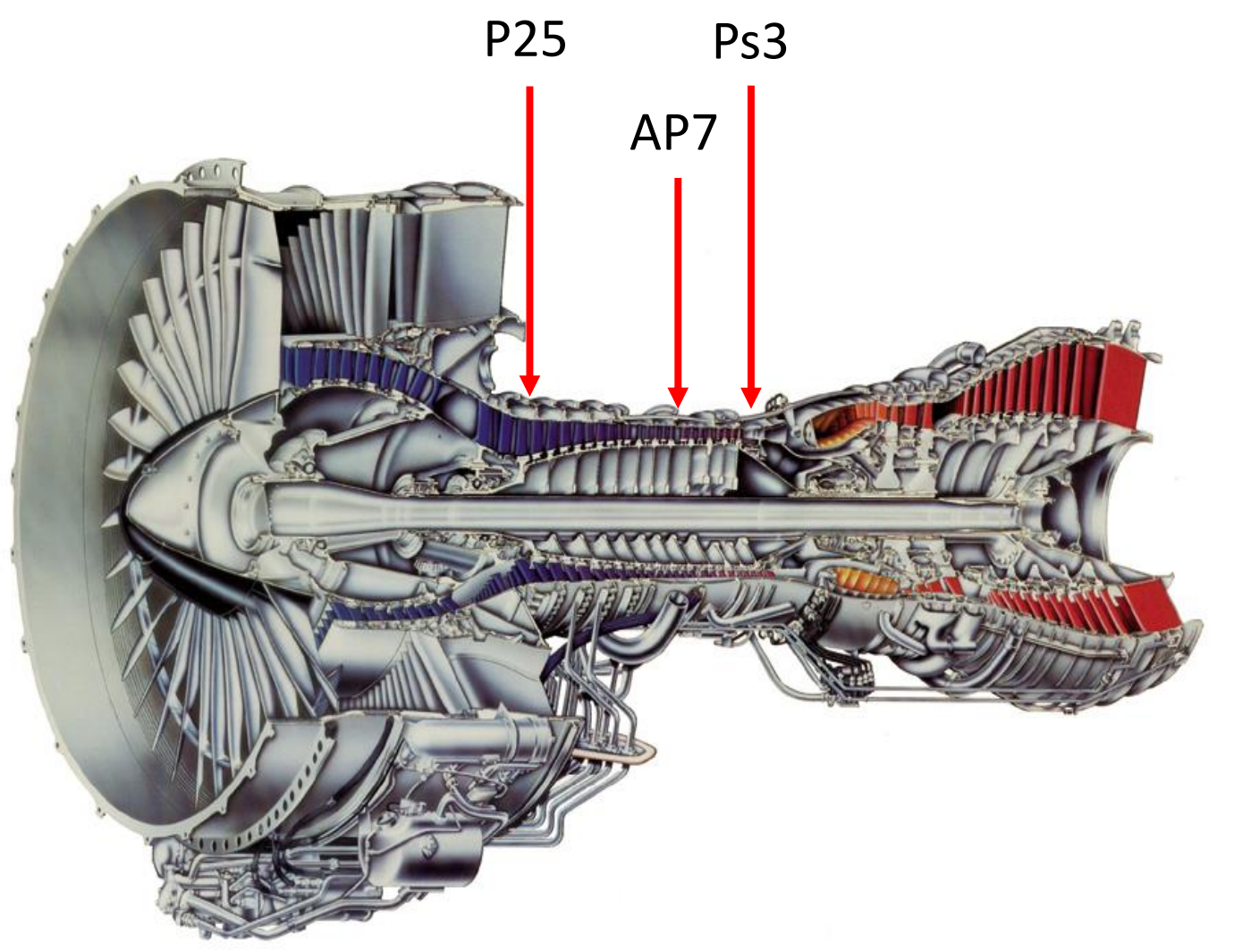

AP7:

High temp capacitive pressure sensor system 


\section{Packaged Sensor On-Engine}

\section{Environmental Health Monitoring Test}

Baseline Engine Test Profile

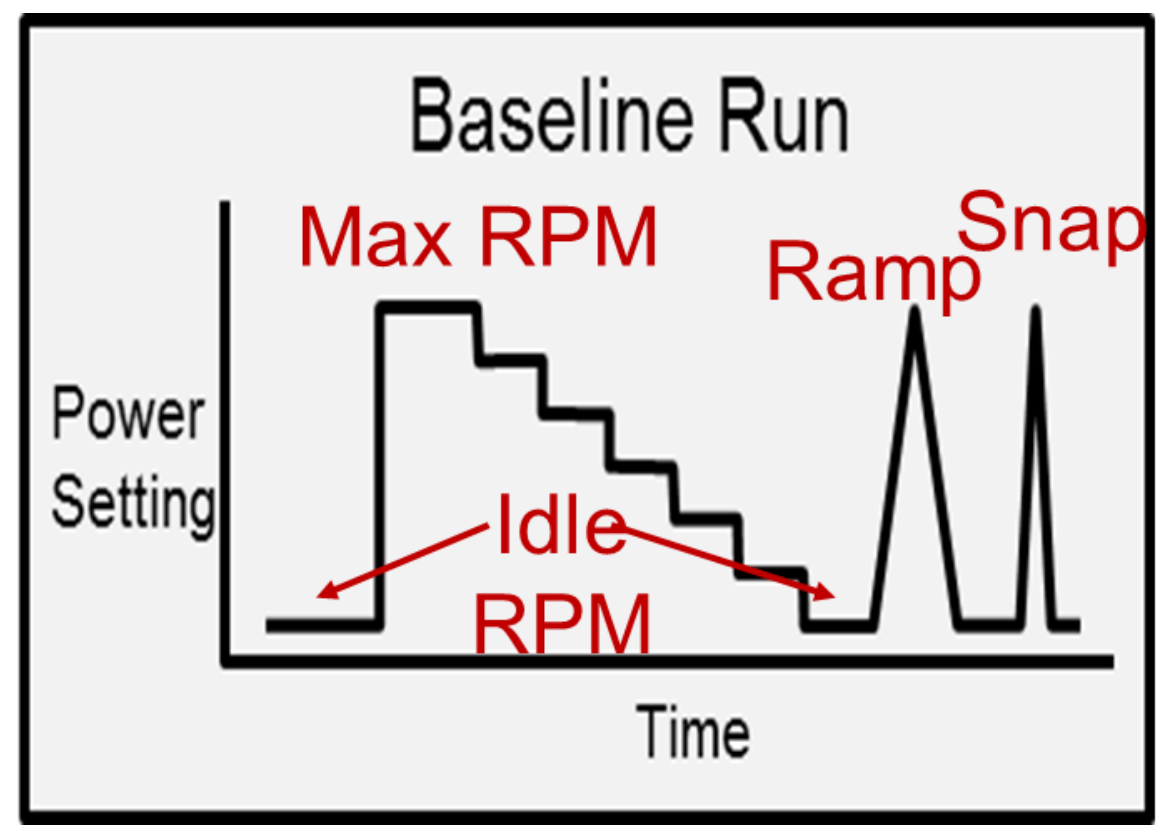

\section{Sensor Output Data}

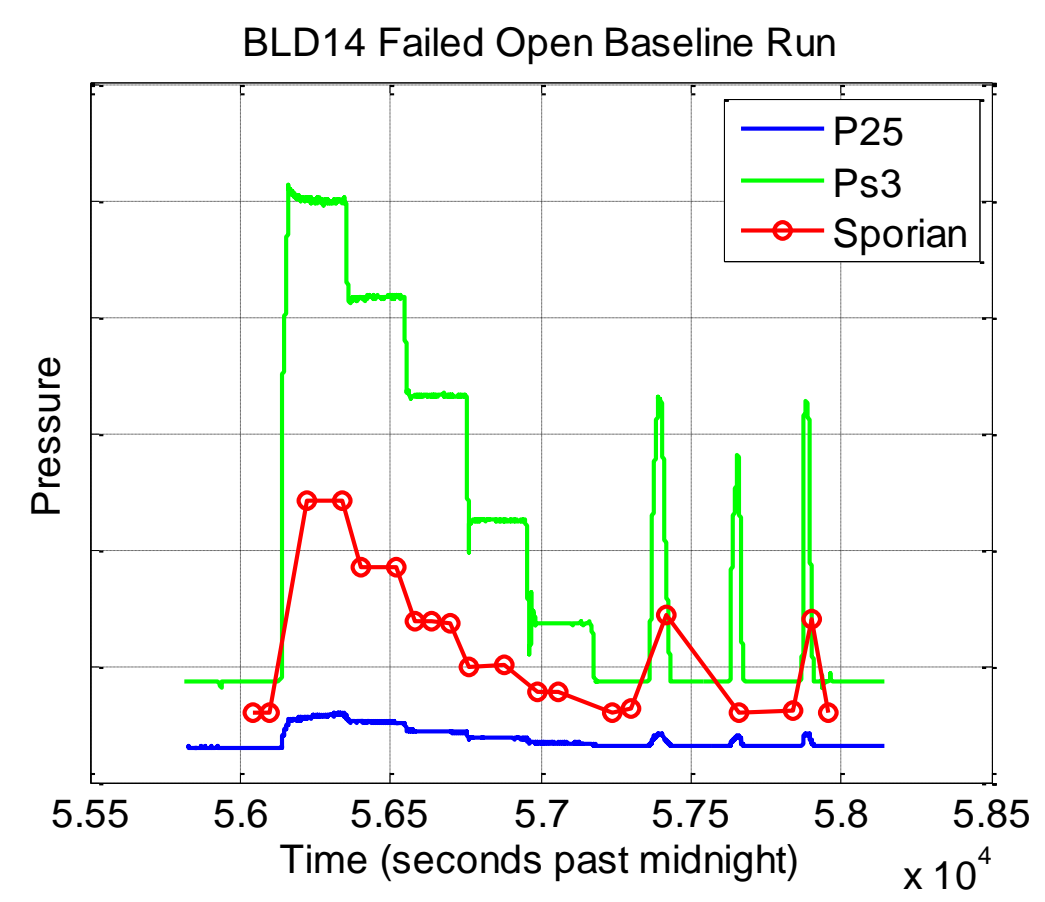




\section{Packaged Sensor On-Engine}

\section{Environmental Health Monitoring Test}

Transient Engine Test Profile

Sensor Output Data
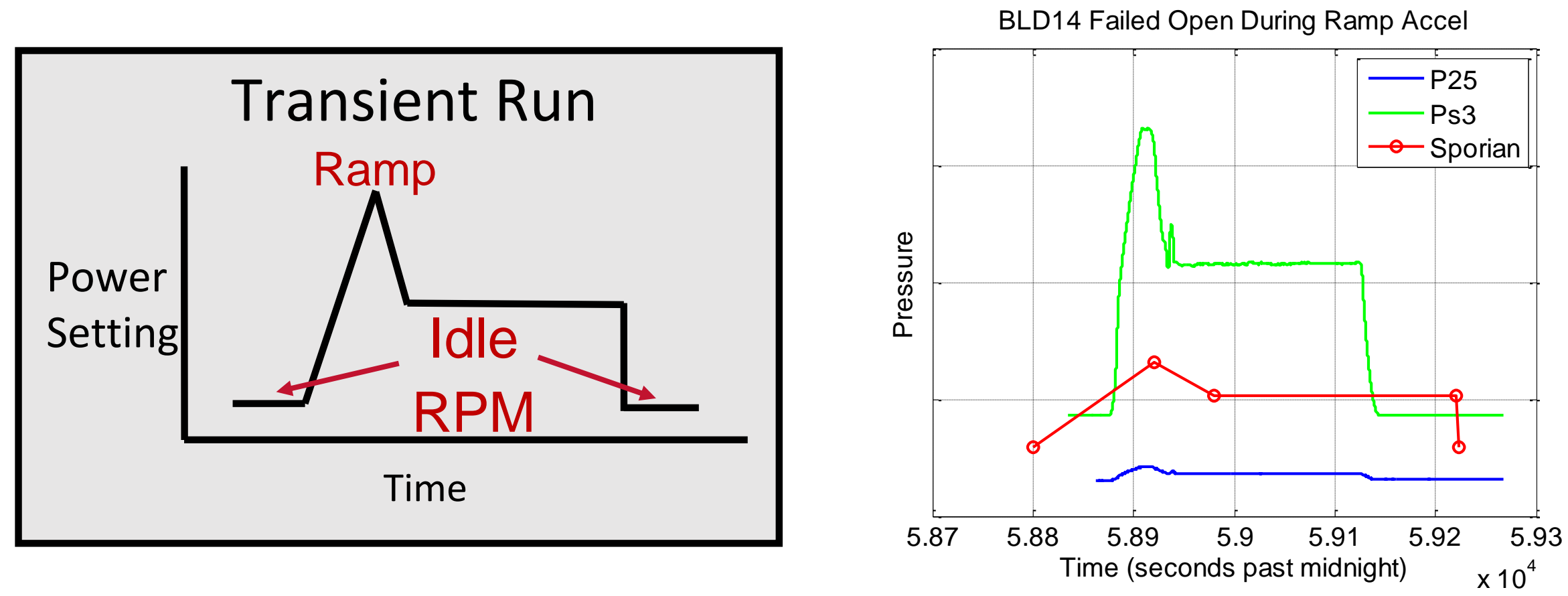


\section{Packaged Sensor On-Engine}

\section{Environmental Health Monitoring Test}

Steady-State Engine

Test Profile

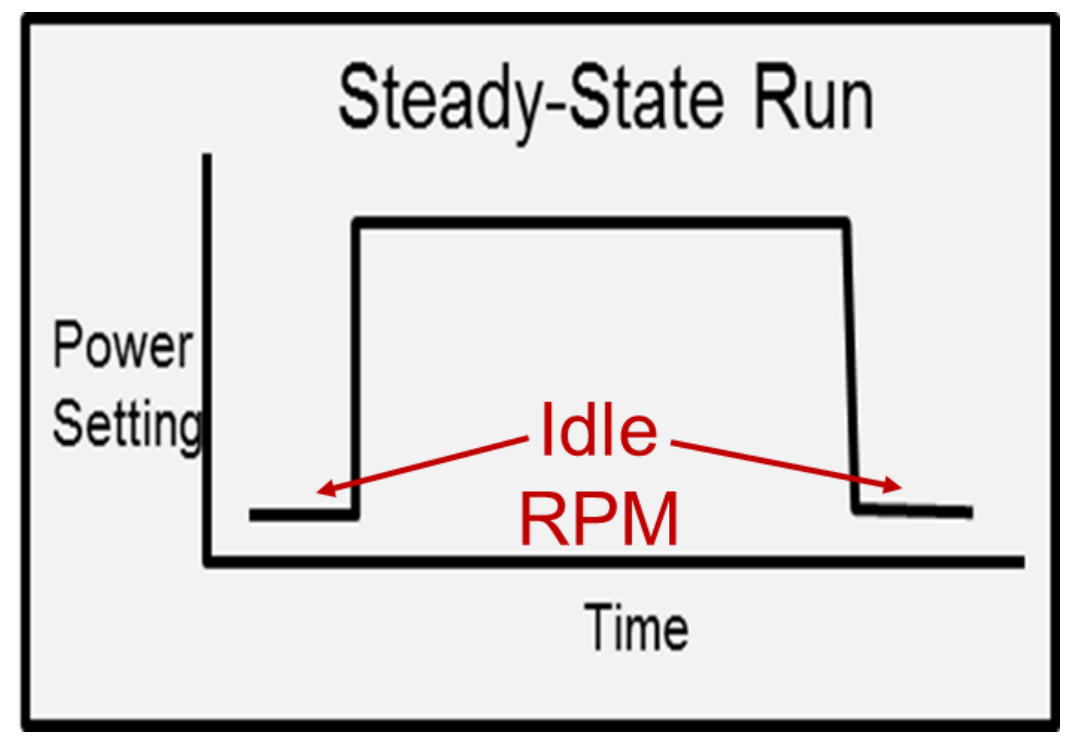

\section{Sensor output data}

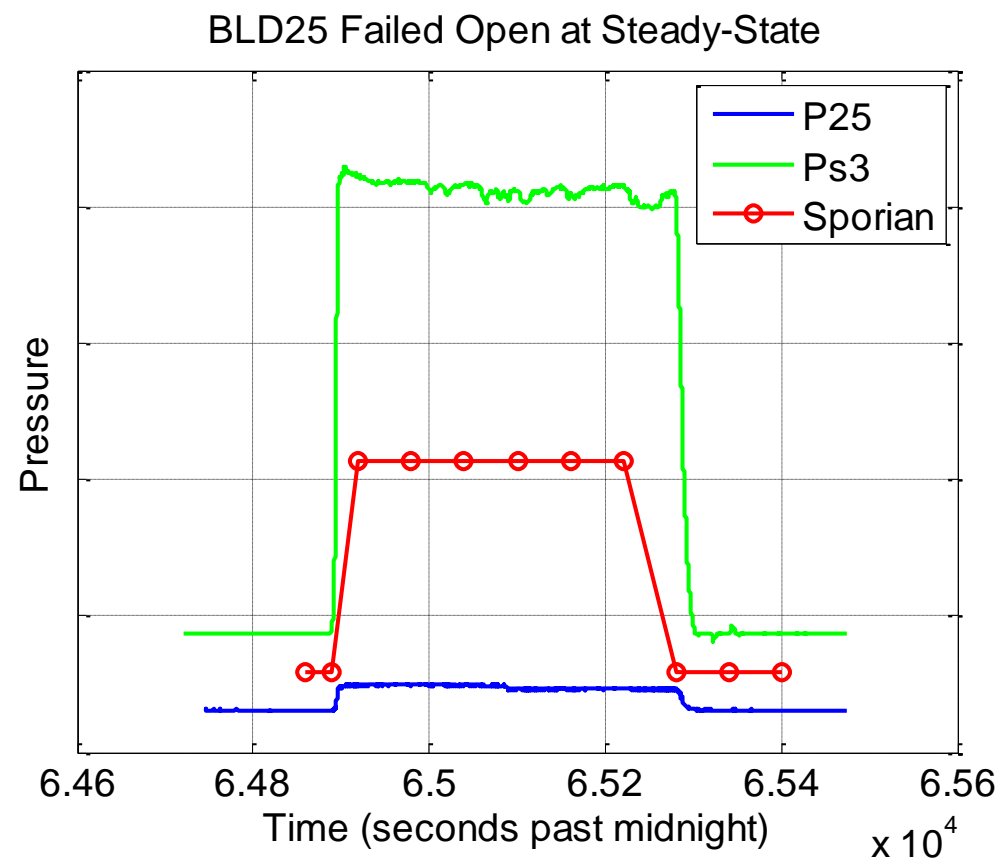




\section{Packaged Sensor On-Engine}

\section{Volcanic Ash Testing}

$1^{\text {st }}$ day of low flow volcanic ash ingestion testing

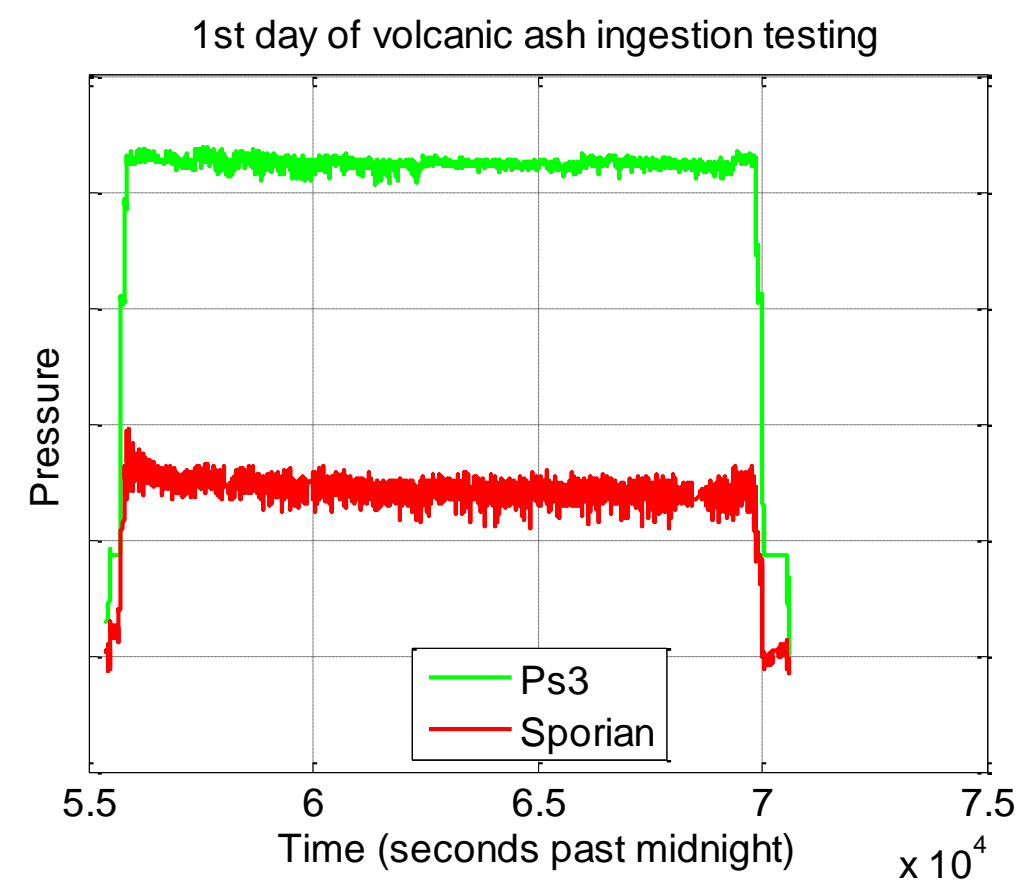

$3^{\text {rd }}$ day of low flow volcanic ash ingestion testing

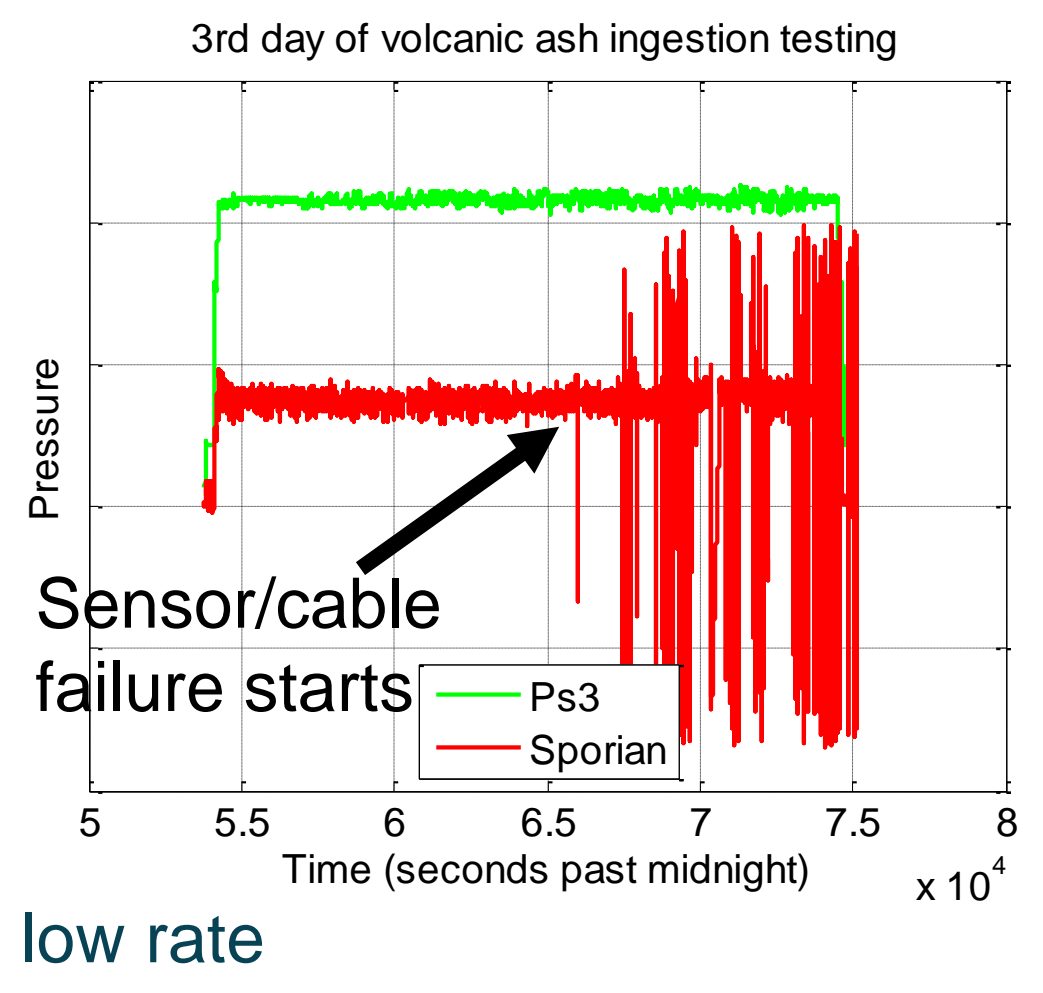

ash testing $1 \mathrm{mg} / \mathrm{cu}$ meter 


\section{Summary}

- Simulated Clapp-type oscillator to prove concept

- Developed a packaged pressure sensor system

- Demonstrated accuracy of simulations vs. measured

- Performed pressure, temperature and vibration acceptance testing

- Successfully demonstrated sensor system tracking engine performance 


\section{Acknowledgements}

NASA Glenn Research Center

Roger Meredith, Elizabeth Mcquaid Jennifer Jordan, Nick Varaljay, Robert Butler, Glenn Beheim and Gary Hunter

\section{Sporian Microsystems}

Keven Harsh, Evan Pilant and Mike Usrey 


\section{Thank you}

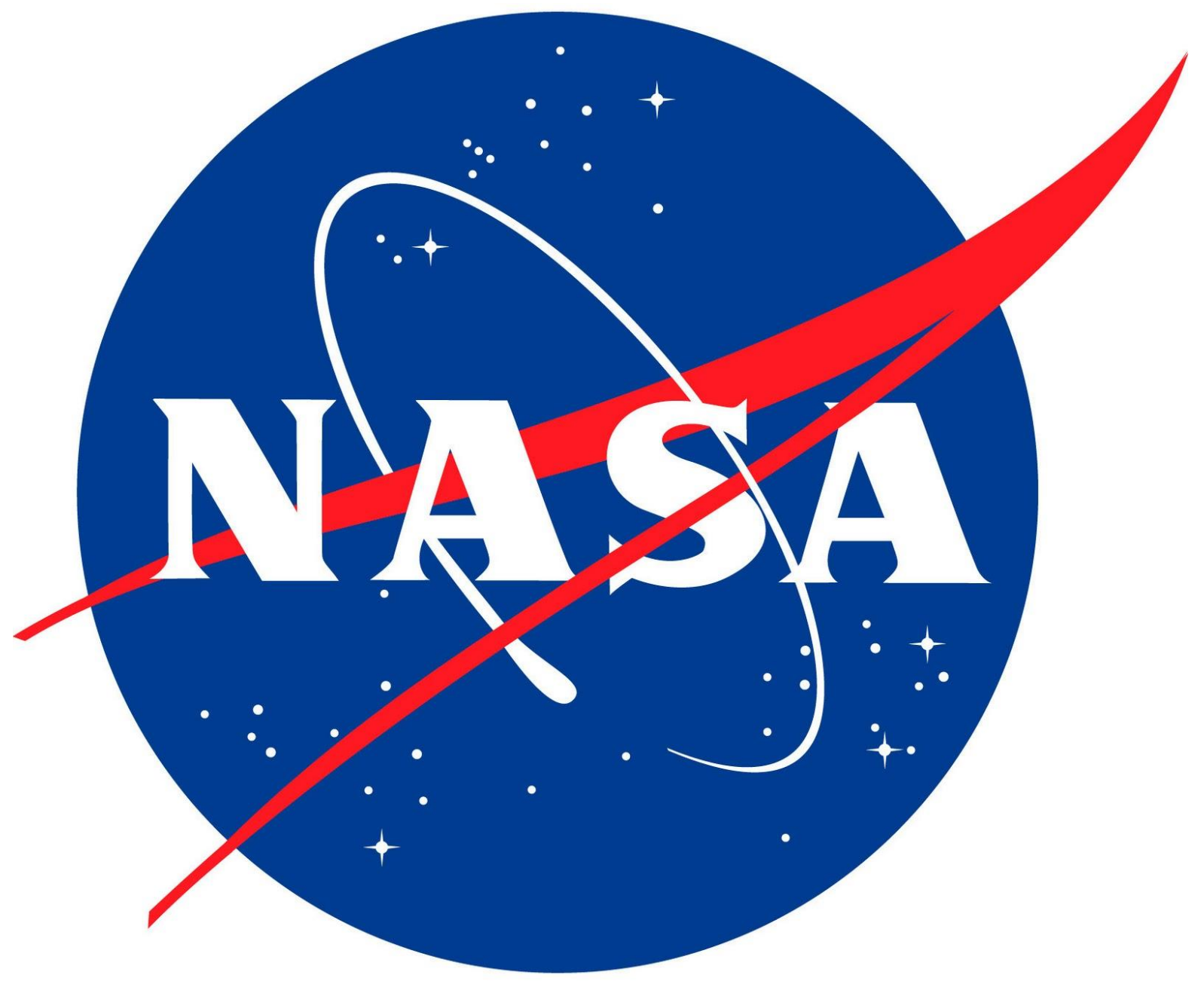




\section{Appendix Slides}




\section{Pressure Sensor System}

\section{Bench-Top Packaged System Characterization Structural Dynamic Testing}
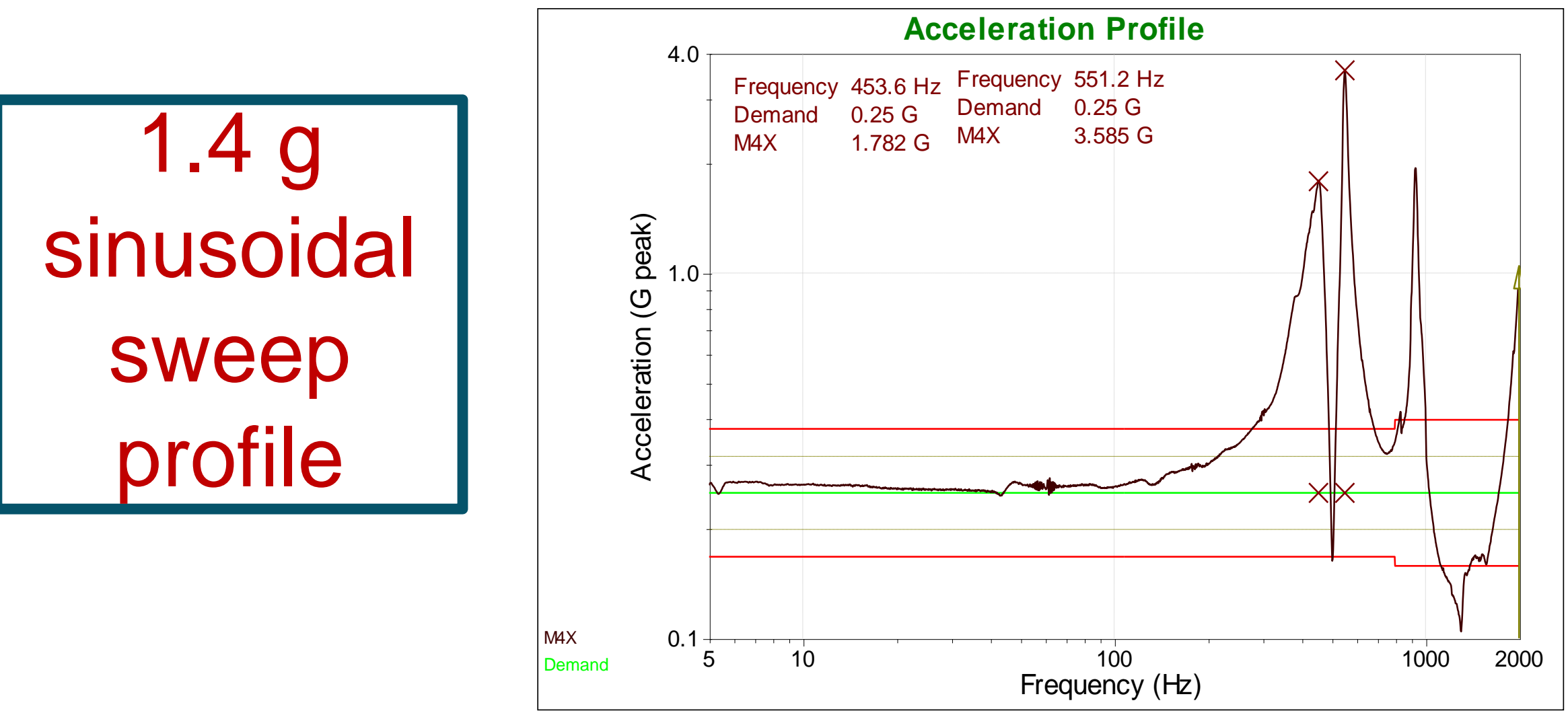


\section{Pressure Sensor System}

\section{Bench-Top Packaged System Characterization}

\section{Structural Dynamic Testing}

\subsection{Grms random vibration profile}

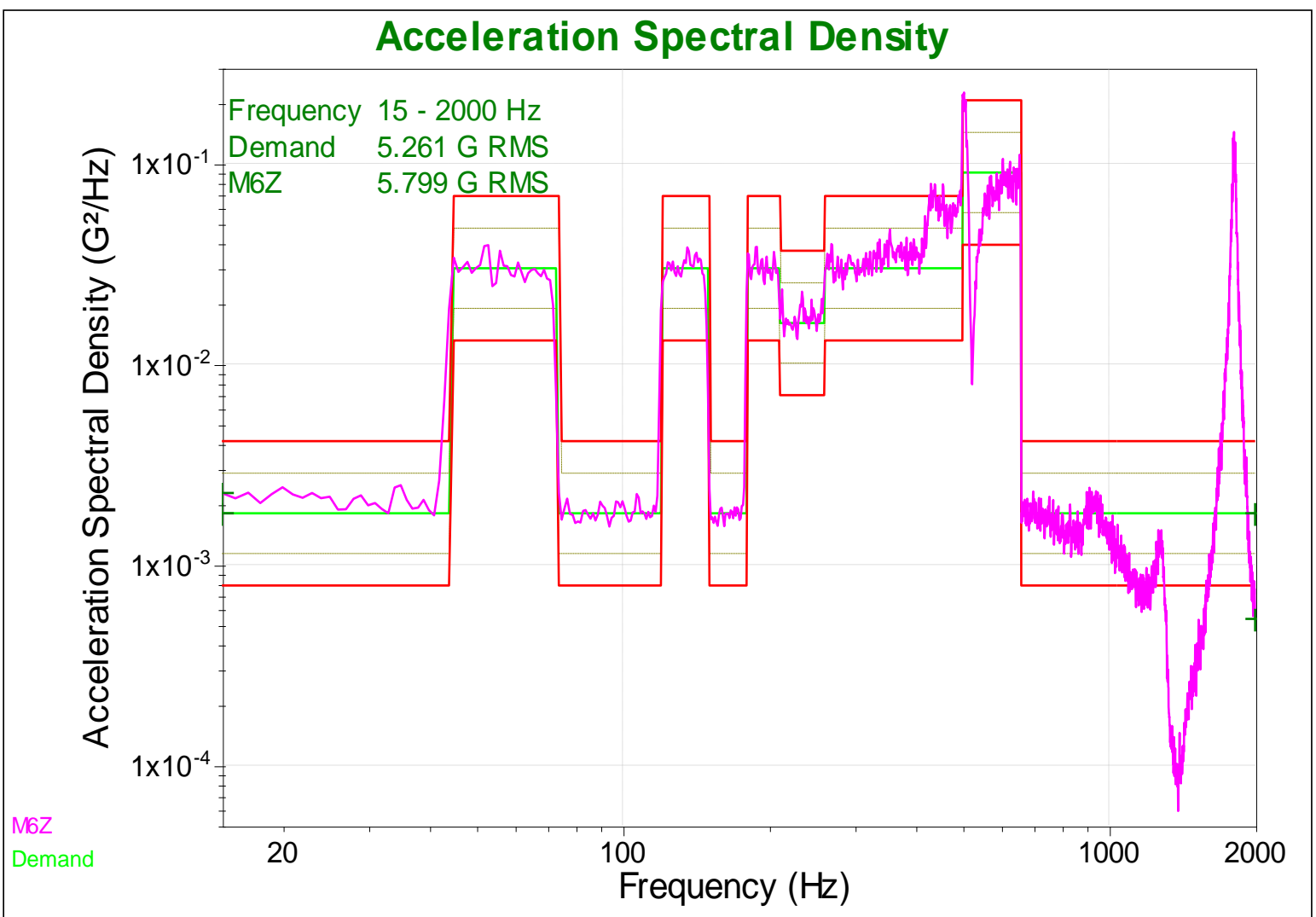

